\title{
A Finite Element Method for the Equations of Motion Arising in Oldroyd Model of Order One with Grad-div Stabilization
}

Bikram Bir

Deepjyoti Goswami ( $\nabla$ deepjyoti@tezu.ernet.in )

https://orcid.org/0000-0002-4300-7750

\section{Research Article}

Keywords: Oldroyd model of order one, grad-div stabilization, backward Euler method, optimal error estimates

Posted Date: February 8th, 2022

DOI: https://doi.org/10.21203/rs.3.rs-1336358/v1

License: (c) (i) This work is licensed under a Creative Commons Attribution 4.0 International License. Read Full License 


\title{
A FINITE ELEMENT METHOD FOR THE EQUATIONS OF MOTION ARISING IN OLDROYD MODEL OF ORDER ONE WITH GRAD-DIV STABILIZATION
}

\author{
Bikram Bir* and Deepjyoti Goswami*
}

\begin{abstract}
In this paper, an inf-sup stable Galerkin mixed finite element method, for the equations of motion arising in the 2D Oldroyd model of order one, with a grad-div stabilization is discussed and an error analysis is carried out. Optimal error estimates for the velocity in $L^{\infty}\left(\mathbf{L}^{2}\right)$-norm and for the pressure in $L^{2}\left(L^{2}\right)$-norm are established in the semidiscrete case. Then, based on backward Euler method, a completely discrete scheme is analyzed, and optimal error estimates are derived. This grad-div stabilization scheme, which adds a stabilization term to the momentum equation, is known to be suitable for high Reynolds number. In conformation with this, the error estimates, in both semi and fully discrete cases, are obtained with constants independent of inverse of viscosity. Finally, we present some numerical results to validate our theoretical results.
\end{abstract}

Key Words. Oldroyd model of order one, grad-div stabilization, backward Euler method, optimal error estimates.

\section{Introduction}

In this paper, we consider a linear viscoelastic model with a memory of past deformation. The model is known as the Oldroyd model of order one [30], and is given by the following integro-differential system:

$$
\frac{\partial \mathbf{u}}{\partial t}+\mathbf{u} \cdot \nabla \mathbf{u}-\mu \Delta \mathbf{u}-\int_{0}^{t} \beta(t-\tau) \Delta \mathbf{u}(\tau) d \tau+\nabla p=\mathbf{f}, \quad \text { in } \Omega, t>0
$$

with incompressibility condition

$$
\nabla \cdot \mathbf{u}=0, \quad \text { on } \Omega, t>0
$$

and initial and boundary conditions

$$
\mathbf{u}(x, 0)=\mathbf{u}_{0} \text { in } \Omega, \quad \mathbf{u}=0, \text { on } \partial \Omega, t \geq 0 .
$$

Here $\Omega$ is a bounded domain in $\mathbb{R}^{2}$ with boundary $\partial \Omega$. And $\mu=\kappa \lambda^{-1}>0$, the kernel $\beta(t)=\gamma \exp (-\delta t), \gamma=$ $\lambda^{-1}\left(\nu-\kappa \lambda^{-1}\right)>0$ and $\delta=\lambda^{-1}>0$, where $\nu>0$ is the kinematic coefficient of viscosity, $\lambda>0$ is the relaxation time and $\kappa>0$ is the retardation time. The unknowns $\mathbf{u}$ and $p$ represent the velocity and the pressure of the fluid, respectively. Further, the forcing term $\mathbf{f}$ and the initial velocity $\mathbf{u}_{0}$ are given functions in their respective domains of definition. This system represents a basic model for polymeric fluids, suspensions or biological fluids; a non-Newtonian model, which has been derived under the assumptions that the material can be regarded as a single stationary macroscopic element with small stress and strain rates, and finds applications in various industries, like, paints, DNA suspensions, biological fluids and some chemical industries. When $\gamma=0$, the system reduces to the well-known Navier-Stokes flows and as such can be considered as an integral perturbation of Navier-Stokes equations. For more details on the mathematical model and physical background, we refer to [30].

Details of early developments of the model and, continuous and semi-discrete cases can be found in $[19,20,34]$ and references therein. And for time discretization, we refer to $[5,35,38]$. There are several other works on this model based on finite element and related framework, for example, see [1,4,27, 29, 40, 41], references there in.

Galerkin mixed finite element for the model has been analyzed on a few occasions [20, 19] with optimal error estimates. However, it is well known that, similar to NSEs, the coupling of velocity and pressure,

\footnotetext{
${ }^{*}$ Department of Mathematical Sciences, Tezpur University, Tezpur, Assam-784028, India. Email: bikramb@tezu.ernet.in, deepjyoti@tezu.ernet.in
} 
through the divergence free term, is in fact not desirable. Methods for decoupling by various means, like penalty method, artificial compressibility method, pressure correction method, projection method etc. attempt to overcome this difficulty by means of artificial conditions. Work in these directions for the Oldroyd model can be found in $[6,27,37,39,42]$. Unfortunately, these methods are less sufficient when Reynolds number is high. This is due to the domination of the advection term on the viscous term, which typically arises for small values of viscosity. It is handled via methods based on stabilization techniques; like streamline upwind/Petrov-Galerkin(SUPG) method, residual-free bubbles enrichment method, local projection stabilization, and interior-penalty methods, see, $[7,9,10,11]$. In particular, in SUPG method, a grad-div stabilization is included which allows to achieve the stability and accuracy for small values of viscosity.

In this paper, we add a grad-div stabilization term to the problem (1.1)-(1.3) and we analyze the effect of this term for high Reynolds number. The main idea is to add a stabilization term with respect to the continuity equation to the momentum equation. It was first proposed by Franca and Hughes [16] to improve the conservation of mass in finite element method. However, the method comes with several other benefits. For example, the use of grad-div stabilization results in improved convergence of preconditioned iteration when the stabilization parameter is too small [31], the well-poseness of the continuous solution as well as the accuracy and convergence of the numerical approximation for small values of viscosity [32], the local mass balance of the system in numerical experiments [13]. Moreover, it has been observed that using grad-div stabilization in the simulation of turbulent flows is sufficient for performing a stable simulation, see [26, fig. 3] and [36, fig. 7].

These observations lead us to the present paper: to derive the error bounds that do not depend on inverse powers of viscosity, for the Galerkin mixed finite element method with grad-div stabilization applied to the Oldroyd model of order one. This is not the first time where similar results have been achieved. In fact, in $[14,15]$, de Frutos et. al. have obtained error bounds with constants independent of inverse powers of viscosity for evolutionary Oseen equations and Navier-Stokes equations, respectively. There are a few more works in this direction for incompressible flow problems but there is no work available for the Oldroyd model of order one to the best our knowledge. In this paper, we extend the analysis of [15] to the Oldroyd model of order one. As in [15], we have carried out our analysis for sufficiently smooth initial data, that is, $\mathbf{u}_{0} \in \mathbf{H}_{0}^{1} \cap \mathbf{H}^{k}(k>2)$, as well as for smooth initial data, $\mathbf{u}_{0} \in \mathbf{H}_{0}^{1} \cap \mathbf{H}^{2}$. But our proofs are shorter and less technically involved than the ones from [15], especially when $\mathbf{u}_{0} \in \mathbf{H}_{0}^{1} \cap \mathbf{H}^{2}$.

We note here that the assumption of sufficiently smooth data comes at a cost of non-local compatibility conditions of various order, for the given data, at time $t=0$. Without these conditions, which do not arise naturally, the solutions of the Oldroyd model of order one can not be assumed to have more than second order derivatives bounded in $L^{2}(\Omega)$ at $t=0$ (see [19]). The analysis for smooth initial data takes into account this lack of regularity at $t=0$.

We would also like to point out that the analysis in both these cases does not differ by much. However the analysis suggests that less regularity of the initial velocity puts a restriction in the order of finite element approximation, when keeping estimates independent of inverse of viscosity. For example, with only smooth initial data, we may get maximum of second order convergence rate in case of velocity, even if we employ higher order approximations, see Remark 3.4.

It is well known that the suitable choice of stabilization parameter for any stabilized scheme is important for accuracy in numerical simulations. In case of grad-div stabilization, a suitable choice of grad-div parameter $\rho$ is shown to be $\mathcal{O}(1)$ for the Navier-Stokes equations and for inf-sup stable finite element pairs, in $[31,33]$. And in [28], it is shown that error can be minimized for $\rho \approx 10^{-1}$. However, larger values of $\rho$ may be needed in special cases, see [17]. A detailed investigation of the choice of grad-div stabilization parameter for steady Stokes problem has been discussed in [25]. They have observed that the choice of grad-div parameter depends on the used norm, the mesh size, the type of mesh, the viscosity, the finite element spaces, and the solution. A similar analysis and numerical simulations have been seen in [3] for the steady state Oseen problem and Navier-Stokes equations.

In this paper, we briefly look into this aspect. Based on the error estimate from Theorem 3.1, we have observed that $\rho=\mathcal{O}(1)$ is a suitable choice for stable mixed finite element spaces. And for MINI element, the choice of $\rho$ can be in the range of $h^{2}$ to 1 , see Remark 3.3. Further, some numerical experiments are carried out. First, we have shown numerically that the grad-div parameter depends on the mesh size, the viscosity and the finite element spaces. Next we have obtained values of grad-div parameter $\rho$ that minimize the $\mathbf{L}^{2}$ and $\mathbf{H}^{1}$ errors for the velocity and $L^{2}$ error for the pressure, for a known solution.

The main results of this article consist of the following:

(i) Optimal error estimates for the velocity in $L^{\infty}\left(\mathbf{L}^{2}\right)$-norm and for the pressure in $L^{2}\left(L^{2}\right)$-norm, where 
the error bounds are independent of the inverse power of viscosity, that is, these results are valid for high Reynolds number.

(ii) Fully discrete optimal error estimates, for the velocity and the pressure, by applying a first-order backward Euler method for temporal discretization. The order of convergence for the velocity in $L^{\infty}\left(\mathbf{L}^{2}\right)$ norm and the pressure in $L^{2}\left(L^{2}\right)$ norm is $\mathcal{O}\left(h^{k}+\Delta t\right)$ when the finite element velocity space and the pressure space are approximated by $k$-th and $(k-1)$-th degree piecewise polynomial, respectively $(k>1)$, where $h$ and $\Delta t$ are the space and time discretization parameter, respectively. These results are valid for high Reynolds number as well.

(iii) Suitable choice of grad-div parameter for stable mixed finite element spaces and for stable equal order spaces like MINI element.

The remaining part of this paper is organised as follows. In Section 2, we introduce the requisite functional spaces and, the assumptions on the domain and on the given data. Then the positivity property of the kernel of the memory term and the Gronwall's lemma, both continuous and discrete versions, are mentioned there. In Section 3, the semidiscrete formulation and error analysis of the stabilized scheme are carried out and in Section 4 backward Euler method is applied to the stabilized system. And finally, in Section 5, some numerical examples are given which conform with our theoretical results. We also obtain numerically suitable values of grad-div parameter, for Oldroyd model of order one, that minimize velocity and pressure errors. Throughout this paper, we will use $C$ as a generic constant, which depends on the given data and not on spatial and time discretization parameters. We note that $C$ may grow exponentially with time, but it does not depend on inverse powers of viscosity.

\section{Preliminaries}

For our subsequent use, we denote by bold face letters the $\mathbb{R}^{2}$-valued function space such as $\mathbf{H}_{0}^{1}=\left[H_{0}^{1}(\Omega)\right]^{2}$, $\mathbf{L}^{2}=\left[L^{2}(\Omega)\right]^{2}$ and $\mathbf{H}^{m}=\left[H^{m}(\Omega)\right]^{2}$. We denote by $\|\cdot\|_{i}$ the usual norm on the Sobolev space $\mathbf{H}^{i}$, for $i=1,2$ and $(\cdot, \cdot)$ and $\|\cdot\|$ be the inner product and norm on $L^{2}$ or $\mathbf{L}^{2}$. The norm on the space of essentially bounded functions $\mathbf{L}^{\infty}(\Omega)$ will be denoted by $\|\cdot\|_{\infty}$. The space $\mathbf{H}_{0}^{1}$ is equipped with the norm

$$
\|\nabla \mathbf{v}\|=\left(\sum_{i, j=1}^{2}\left(\partial_{j} v_{i}, \partial_{j} v_{i}\right)\right)^{1 / 2}=\left(\sum_{i=1}^{2}\left(\nabla v_{i}, \nabla v_{i}\right)\right)^{1 / 2}
$$

We denote by $\mathbf{J}_{1}$ and $\mathbf{J}$, the divergence free subspaces of $\mathbf{H}_{0}^{1}$ and $\mathbf{L}^{2}$, respectively.

$$
\begin{aligned}
\mathbf{J}_{1} & =\left\{\boldsymbol{\phi} \in \mathbf{H}_{0}^{1}: \nabla \cdot \boldsymbol{\phi}=0\right\} . \\
\mathbf{J}=\left\{\phi \in \mathbf{L}^{2}: \nabla \cdot \boldsymbol{\phi}\right. & \left.=0 \text { in } \Omega,\left.\boldsymbol{\phi} \cdot \mathbf{n}\right|_{\partial \Omega}=0 \text { holds weakly }\right\}
\end{aligned}
$$

where $\mathbf{n}$ is the outward normal to the boundary $\partial \Omega$ and $\left.\boldsymbol{\phi} \cdot \mathbf{n}\right|_{\partial \Omega}=0$ should be understood in the sense of trace in $\mathbf{H}^{-1 / 2}(\partial \Omega)$. Let $H^{m} / \mathbb{R}$ be the quotient space with norm $\|\phi\|_{H^{m} / \mathbb{R}}=\inf _{c \in \mathbb{R}}\|\phi+c\|_{m}$. For $m=0$, it is denoted by $L^{2} / \mathbb{R}$. For any Banach space $X$, let $L^{p}(0, T ; X)$ denote the space of measurable $X$-valued functions $\boldsymbol{\phi}$ on $(0, T)$ such that

$$
\int_{0}^{T}\|\phi(t)\|_{X}^{p} d t<\infty, \quad \text { if } 1 \leq p<\infty, \quad \text { and } \quad \underset{0<t<T}{\operatorname{ess} \sup }\|\phi(t)\|_{X}<\infty, \quad \text { if } p=\infty .
$$

The dual space of $H^{m}(\Omega)$, denoted by $H^{-m}(\Omega)$, is defined as the completion of $C^{\infty}(\bar{\Omega})$ with respect to the norm

$$
\|\phi\|_{-m}:=\sup \left\{\frac{(\phi, \psi)}{\|\psi\|_{m}}: \psi \in H^{m}(\Omega),\|\psi\|_{m} \neq 0\right\} .
$$

The following Sobolev's embedding [2] will be used for our analysis: For $1 \leq p \leq d / s$, let $q$ be such that $\frac{1}{q}=\frac{1}{p}-\frac{s}{d}$, then there exists a constant $C$ independent of $s$, such that

$$
\|v\|_{L^{q^{\prime}}(\Omega)} \leq C\|v\|_{W^{s, p}(\Omega)}, \quad \frac{1}{q^{\prime}} \geq \frac{1}{q}, \quad v \in W^{s, p}(\Omega) .
$$


If $p>\frac{d}{s}$ the above result is valid for $q^{\prime}=\infty$. In our case we consider $d=2$. The similar embedding inequality holds for vector-valued functions. Through out this paper, we make the following assumption:

(A1) For $\mathbf{g} \in \mathbf{H}^{m-1}$ with $m \geq 1$, let the unique pair of solutions $\left\{\mathbf{v} \in \mathbf{J}_{1}, q \in L^{2} / \mathbb{R}\right\}$ for the steady state Stokes problem

$$
-\Delta \mathbf{v}+\nabla q=\mathbf{g}, \quad \nabla \cdot \mathbf{v}=0 \quad \text { in } \Omega ;\left.\quad \mathbf{v}\right|_{\partial \Omega}=0
$$

satisfy the following regularity result [23]:

$$
\|\mathbf{v}\|_{m+1}+\|q\|_{H^{m} / \mathbb{R}} \leq C\|\mathbf{g}\|_{m-1} .
$$

We first note here that (A1) implies (see [22])

$$
\|\mathbf{v}\|_{2} \leq C\|\tilde{\Delta} \mathbf{v}\|, \quad \forall \mathbf{v} \in \mathbf{J}_{1} \cap \mathbf{H}^{2}, \quad\|\mathbf{v}\| \leq \lambda_{1}^{-1 / 2}\|\mathbf{v}\|_{1}, \quad \mathbf{v} \in \mathbf{H}_{0}^{1}, \quad\|\mathbf{v}\|_{1} \leq \lambda_{1}^{-1 / 2}\|\mathbf{v}\|_{2}, \quad \mathbf{v} \in \mathbf{J}_{1} \cap \mathbf{H}^{2},
$$

where $\tilde{\Delta}=P \Delta, \tilde{\Delta}: \mathbf{J}_{1} \cap \mathbf{H}^{2} \subset \mathbf{J} \rightarrow \mathbf{J}$ is the Stokes operator and $P$ is the orthogonal projection of $\mathbf{L}^{2}$ onto $\mathbf{J}$. And $\lambda_{1}>0$ is the least positive eigenvalue of $\tilde{\Delta}$.

We will subsequently use the Gagliardo-Nirenberg inequality [24]

$$
\|\phi\|_{L^{p}} \leq C\|\phi\|^{2 / p}\|\nabla \phi\|^{1-2 / p}, \quad \forall \boldsymbol{\phi} \in \mathbf{H}_{0}^{1},
$$

where $2 \leq p<\infty$ and $C=C(p, \Omega)$. Also, we will consider the Agmon's inequality [24]

$$
\|\phi\|_{L^{\infty}} \leq C\|\phi\|^{1 / 2}\|\Delta \phi\|^{1 / 2}, \quad \forall \phi \in \mathbf{H}^{2},
$$

where $C=C(\Omega)$.

Remark 2.1. We will use the discrete version of the above two inequalities with constants uniform in discretizing parameter $h$, following [24].

We now make the following assumption about the given data for the problem (1.1)-(1.3).

(A2) The external force $\mathbf{f}$ satisfy for some $M_{0}>0$ and for $0<T \leq \infty$

$$
D_{t}^{l} \mathbf{f} \in L^{\infty}\left(0, T ; \mathbf{H}^{m}\right) \quad \text { with } \sup _{0<t<T}\left\{\left\|D_{t}^{l} \mathbf{f}\right\|_{m}\right\} \leq M_{0} \text {, for some integer } m \geq 0 \text { and } l \geq 0 .
$$

Before going into the details, we define the continuous bilinear form $a(\cdot, \cdot)$ on $\mathbf{H}_{0}^{1} \times \mathbf{H}_{0}^{1}$ by

$$
a(\mathbf{v}, \mathbf{w})=(\nabla \mathbf{v}, \nabla \mathbf{w}), \quad \forall \mathbf{v}, \mathbf{w} \in \mathbf{H}_{0}^{1},
$$

and the continuous trilinear form $b(\cdot, \cdot, \cdot)$ on $\mathbf{H}_{0}^{1} \times \mathbf{H}_{0}^{1} \times \mathbf{H}_{0}^{1}$ by

$$
b(\mathbf{v}, \mathbf{w}, \boldsymbol{\phi})=((\mathbf{v} \cdot \nabla) \mathbf{w}, \boldsymbol{\phi})+\frac{1}{2}((\nabla \cdot \mathbf{v}) \mathbf{w}, \boldsymbol{\phi})=\frac{1}{2}((\mathbf{v} \cdot \nabla) \mathbf{w}, \boldsymbol{\phi})-\frac{1}{2}((\mathbf{v} \cdot \nabla) \boldsymbol{\phi}, \mathbf{w}), \quad \forall \mathbf{v}, \mathbf{w}, \boldsymbol{\phi} \in \mathbf{H}_{0}^{1} .
$$

It is clearly seen that

$$
b(\mathbf{v}, \mathbf{w}, \boldsymbol{\phi})=-b(\mathbf{v}, \boldsymbol{\phi}, \mathbf{w}), \quad \forall \mathbf{v}, \mathbf{w}, \boldsymbol{\phi} \in \mathbf{H}_{0}^{1} .
$$

In particular

$$
b(\mathbf{v}, \mathbf{w}, \mathbf{w})=0, \quad \forall \mathbf{v}, \mathbf{w} \in \mathbf{H}_{0}^{1} .
$$

Let us introduce the weak formulation of (1.1)-(1.3): To find a pair of functions $\{\mathbf{u}(t), p(t)\} \in \mathbf{H}_{0}^{1} \times L^{2} / \mathbb{R}, t>$ 0 such that for $\phi \in \mathbf{H}_{0}^{1}, \chi \in L^{2}$

$$
\left\{\begin{array}{l}
\left(\mathbf{u}_{t}, \boldsymbol{\phi}\right)+\mu a(\mathbf{u}, \boldsymbol{\phi})+b(\mathbf{u}, \mathbf{u}, \boldsymbol{\phi})+\int_{0}^{t} \beta(t-s) a(\mathbf{u}(s), \boldsymbol{\phi}) d s=(p, \nabla \cdot \boldsymbol{\phi})+(\mathbf{f}, \boldsymbol{\phi}), \\
(\nabla \cdot \mathbf{u}, \chi)=0 .
\end{array}\right.
$$

Equivalently, find $\mathbf{u}(t) \in \mathbf{J}_{1}, t>0$ such that

$$
\left(\mathbf{u}_{t}, \boldsymbol{\phi}\right)+\mu a(\mathbf{u}, \boldsymbol{\phi})+b(\mathbf{u}, \mathbf{u}, \boldsymbol{\phi})+\int_{0}^{t} \beta(t-s) a(\mathbf{u}(s), \boldsymbol{\phi}) d s=(\mathbf{f}, \boldsymbol{\phi}), \quad \forall \boldsymbol{\phi} \in \mathbf{J}_{1} .
$$


For existence and uniqueness of the problem (2.4) and (2.5), we refer to [19]. And for the regularity of the solutions $\mathbf{u}$ and $p$, we make the following assumptions depending on whether the initial velocity $\mathbf{u}_{0}$ is sufficiently smooth or just smooth:

(A3) Let us assume that $\mathbf{u}_{0} \in \mathbf{H}^{\max \{2, k\}}$ is sufficiently smooth and the solution pair (u, $p$ ) of (2.4) satisfies

$$
\mathbf{u} \in L^{2}\left(0, T ; \mathbf{H}^{k+1}\right) \cap L^{2}\left(0, T ;\left(W^{1, \infty}(\Omega)\right)^{2}\right) \cap L^{\infty}\left(0, T ; \mathbf{H}^{k}\right), p \in L^{2}\left(0, T ; \mathbf{H}^{k} / \mathbb{R}\right)
$$

for all $k \geq 1$. Further, there exists a positive constant $C$ that does not depend on the inverse power of $\mu$ such that for all $k \geq 1$, the following hold:

$$
\begin{gathered}
\max _{0 \leq t \leq T}\left(\|\mathbf{u}(t)\|_{k}^{2}+\|p(t)\|_{H^{k-1} / \mathbb{R}}^{2}\right) \leq C, \quad \int_{0}^{t}\|\nabla \mathbf{u}(s)\|_{\infty}^{2} d s \leq C t \\
e^{-2 \alpha t} \int_{0}^{t} e^{2 \alpha s}\left(\|\mathbf{u}(s)\|_{k+1}^{2}+\left\|\mathbf{u}_{s}(s)\right\|_{k-1}^{2}+\|p(s)\|_{H^{k} / \mathbb{R}}^{2}\right) d s \leq C .
\end{gathered}
$$

(A3') Let us assume that the initial data $\mathbf{u}_{0} \in \mathbf{J}_{1} \cap \mathbf{H}^{2}$ is smooth and the solution pair ( $\left.\mathbf{u}, p\right)$ of (2.4) satisfies

$$
(\tau(t))^{k-2} \mathbf{u} \in L^{2}\left(0, T ; \mathbf{H}^{k+1}\right) \cap L^{\infty}\left(0, T ; \mathbf{H}^{k}\right),(\tau(t))^{k-2} p \in L^{2}\left(0, T ; \mathbf{H}^{k} / \mathbb{R}\right), \mathbf{u} \in L^{2}\left(0, T ;\left(W^{1, \infty}(\Omega)\right)^{2}\right)
$$

for $k \geq 2$ and for $k=1$,

$$
\mathbf{u} \in L^{2}\left(0, T ; \mathbf{H}^{2}\right) \cap L^{2}\left(0, T ;\left(W^{1, \infty}(\Omega)\right)^{2}\right) \cap L^{\infty}\left(0, T ; \mathbf{H}^{1}\right), p \in L^{2}\left(0, T ; \mathbf{H}^{1} / \mathbb{R}\right) .
$$

Further, there exists a positive constant $C$ that does not depend on the inverse power of $\mu$ such that, the following hold: For $k=1$,

$$
\int_{0}^{t}\|\nabla \mathbf{u}(s)\|_{\infty}^{2} d s \leq C t, \quad \max _{0 \leq t \leq T}\|\mathbf{u}(t)\|_{1}^{2}+e^{-2 \alpha t} \int_{0}^{t} e^{2 \alpha s}\|\mathbf{u}(s)\|_{2}^{2} d s \leq C .
$$

For $k \geq 2$, and for $\tau(t)=\min \{1, t\}$

$$
\begin{array}{r}
\max _{0 \leq t \leq T}(\tau(t))^{k-2}\left(\|\mathbf{u}(t)\|_{k}^{2}+\|p(t)\|_{H^{k-1} / \mathbb{R}}^{2}\right) \leq C, \\
e^{-2 \alpha t} \int_{0}^{t} e^{2 \alpha s}(\tau(s))^{k-2}\left(\|\mathbf{u}(s)\|_{k+1}^{2}+\left\|\mathbf{u}_{s}(s)\right\|_{k-1}^{2}+\|p(s)\|_{H^{k} / \mathbb{R}}^{2}\right) d s \leq C .
\end{array}
$$

Before we move to the next section, we present below a few lemmas which will be used in our subsequent analysis. First one deals with the positivity of the kernel $\beta$. The result is borrowed from [34, Lemma 2.1].

Lemma 2.1. For arbitrary $\alpha>0, t^{*}>0$ and $\phi \in L^{2}\left(0, t^{*}\right)$, the following positive definite property holds

$$
\int_{0}^{t^{*}}\left(\int_{0}^{t} \exp [-\alpha(t-s)] \phi(s) d s\right) \phi(t) d t \geq 0 .
$$

Second and third Lemmas are on Gronwall's inequality.

Lemma 2.2 (Gronwall's Lemma). Let $g, h, y$ be three locally integrable non-negative functions on the time interval $[0, \infty)$ such that for all $t \geq 0$

$$
y(t)+G(t) \leq C+\int_{0}^{t} h(s) d s+\int_{0}^{t} g(s) y(s) d s,
$$

where $G(t)$ is a non-negative function on $[0, \infty)$ and $C \geq 0$ is a constant. Then,

$$
y(t)+G(t) \leq\left(C+\int_{0}^{t} h(s) d s\right) \exp \left(\int_{0}^{t} g(s) d s\right) .
$$

Lemma 2.3 (discrete Gronwall's Lemma [23]). Let $k, B$ and $\left\{a_{i}, b_{i}, c_{i}, d_{i}\right\}_{i \in \mathbb{N}}$ be non-negative numbers such that

$$
a_{n}+k \sum_{i=1}^{n} b_{i} \leq B+k \sum_{i=1}^{n} c_{i}+k \sum_{i=1}^{n} d_{i} a_{i}, n \geq 1
$$


Suppose that $k d_{i} \leq 1$, for all $i$. Set $\gamma_{i}=\left(1-k d_{i}\right)^{-1}$. Then,

$$
a_{n}+k \sum_{i=1}^{n} b_{i} \leq\left\{B+k \sum_{i=1}^{n} c_{i}\right\} \exp \left(k \sum_{i=1}^{n} \gamma_{i} d_{i}\right) .
$$

If the last sum of (2.6) extends only up to $n-1$, then the estimate (2.7) holds for all $k>0$, with $\gamma_{i} \equiv 1$.

Remark 2.2. Whenever we use discrete Gronwall's Lemma, it restricts the time step size $k$.

\section{Galerkin Finite Element Method}

In this section, we consider the finite element Galerkin approximations to the problem (1.1)-(1.3) with graddiv stabilization. From now on, we denote $h$, with $0<h<1$, to be a real positive spatial discretization parameter, tending to zero. Let $\mathcal{T}_{h}$ be a finite decomposition of mesh size $h$, of the polygonal domain $\bar{\Omega}$ into closed subsets, triangles or quadrilaterals in two dimension. The decomposition $\mathcal{T}_{h}$ is assumed to be "face to face" and satisfy a "uniform size" condition:

Any two elements of $\mathcal{T}_{h}$ meet only in entire common sides or in vertices. Each element of $\mathcal{T}_{h}$ contains a circle of radius $\kappa_{1} h$ and its contained in a circle of radius $\kappa_{2} h$, these constant $\kappa_{1}, \kappa_{2}$ being independent of $h$.

Let $\mathbf{H}_{h}$ and $L_{h}$ be two families of finite element spaces, finite dimensional subspaces of $\mathbf{H}_{0}^{1}$ and $L^{2} / \mathbb{R}$, respectively, approximating the velocity vector and the pressure. It is assumed that the spaces $\left(\mathbf{H}_{h}, L_{h}\right)$ are of the form $\left(P_{k}, P_{k-1}\right)$ where $P_{k}$ comprises of piecewise polynomial of degree at most $k, k>1$. [However for $k=1$, we consider the mini element $\left(P_{1} b, P_{1}\right)$ where $P_{1} b$ is the $P_{1}$ space with bubble enrichment.]

Assume that the following approximation properties are satisfied for the spaces $\mathbf{H}_{h}$ and $L_{h}$ :

(B1) For each $\mathbf{w} \in \mathbf{H}_{0}^{1} \cap \mathbf{H}^{k+1}$ and $q \in H^{k} / \mathbb{R}$ with $k \geq 1$, then there exist approximations $i_{h} w \in \mathbf{H}_{h}$ and $j_{h} q \in L_{h}$ such that

$$
\left\|\mathbf{w}-i_{h} \mathbf{w}\right\|+h\left\|\nabla\left(\mathbf{w}-i_{h} \mathbf{w}\right)\right\| \leq C h^{j+1}\|\mathbf{w}\|_{j+1}, \quad\left\|q-j_{h} q\right\| \leq C h^{j}\|q\|_{j}, \quad 0 \leq j \leq k
$$

Further, we will assume that the meshes are quasi-uniform and the following inverse hypothesis holds for $\mathbf{v}_{h} \in \mathbf{H}_{h}$, see [12, Theorem 3.2.6]

$$
\left\|\mathbf{v}_{h}\right\|_{W^{m, p}(K)^{d}} \leq C h^{n-m-d\left(\frac{1}{q}-\frac{1}{p}\right)}\left\|\mathbf{v}_{h}\right\|_{W^{n, q}(K)^{d}},
$$

where $0 \leq n \leq m \leq 1,0 \leq q \leq p \leq \infty, h$ be the diameter of the mesh cell $K \in \mathcal{T}_{h}$ and $\|\cdot\|_{W^{m, p}(K)^{d}}$ is the norm in Sobolev space $W^{m, p}(K)^{d}$.

Now, we consider the discrete analogue of the weak formulations (1.1)-(1.3) with a grad-div stabilization term: Find $\left(\mathbf{u}_{h}, p_{h}\right)$ in $\mathbf{H}_{h} \times L_{h}$ satisfying

$$
\left\{\begin{array}{c}
\left(\mathbf{u}_{h t}, \boldsymbol{\phi}_{h}\right)+\mu a\left(\mathbf{u}_{h}, \boldsymbol{\phi}_{h}\right)+b\left(\mathbf{u}_{h}, \mathbf{u}_{h}, \boldsymbol{\phi}_{h}\right)+\int_{0}^{t} \beta(t-\tau) a\left(\mathbf{u}_{h}(\tau), \boldsymbol{\phi}_{h}\right) d \tau \\
-\left(p_{h}, \nabla \cdot \boldsymbol{\phi}_{h}\right)+\rho\left(\nabla \cdot \mathbf{u}_{h}, \nabla \cdot \boldsymbol{\phi}_{h}\right)=\left(\mathbf{f}, \boldsymbol{\phi}_{h}\right), \quad \forall \boldsymbol{\phi}_{h} \in \mathbf{H}_{h}, \\
\left(\nabla \cdot \mathbf{u}_{h}, \chi_{h}\right)=0, \quad \forall \chi_{h} \in L_{h},
\end{array}\right.
$$

where $\rho \geq 0$ is the stabilization parameter and $\mathbf{u}_{0 h}=P_{h} \mathbf{u}_{0} \in \mathbf{H}_{h}$ is suitable approximation of $\mathbf{u}_{0} \in \mathbf{J}_{1}$. Let us consider the associated weekly divergence free subspace $\mathbf{J}_{h}$ of the discrete space $\mathbf{H}_{h}$ as

$$
\mathbf{J}_{h}=\left\{\mathbf{v}_{h} \in \mathbf{H}_{h}:\left(\chi_{h}, \nabla \cdot \mathbf{v}_{h}\right)=0, \forall \chi_{h} \in L_{h}\right\}
$$

Note that the space $\mathbf{J}_{h}$ is not a subspace of $\mathbf{J}_{1}$. As $\mathbf{J}_{h}$ as above, we now introduce an equivalent Galerkin approximation as: Find $\mathbf{u}_{h}(t) \in \mathbf{J}_{h}$ such that $\mathbf{u}_{h}(0)=\mathbf{u}_{0 h}$ and for $t>0$

$$
\left(\mathbf{u}_{h t}, \phi_{h}\right)+\mu a\left(\mathbf{u}_{h}, \phi_{h}\right)+b\left(\mathbf{u}_{h}, \mathbf{u}_{h}, \phi_{h}\right)+\int_{0}^{t} \beta(t-\tau) a\left(\mathbf{u}_{h}(\tau), \phi_{h}\right) d \tau+\rho\left(\nabla \cdot \mathbf{u}_{h}, \nabla \cdot \boldsymbol{\phi}_{h}\right)=\left(\mathbf{f}, \boldsymbol{\phi}_{h}\right)
$$

for all $\phi_{h} \in \mathbf{J}_{h}$. Below, we present a priori estimate for the discrete solution.

Lemma 3.1. The following stability estimate holds for the discrete velocity for all $0 \leq t \leq T, T>0$

$$
\left\|\mathbf{u}_{h}(t)\right\|^{2}+2 e^{-2 \alpha t} \int_{0}^{t} e^{2 \alpha s}\left(\mu\left\|\nabla \mathbf{u}_{h}(s)\right\|^{2}+\rho\left\|\nabla \cdot \mathbf{u}_{h}(s)\right\|^{2}\right) d s \leq\left(e^{-2 \alpha t}\left\|\mathbf{u}_{0 h}\right\|^{2}+\frac{\|\mathbf{f}\|_{\infty}^{2}}{2 \alpha}\right) e^{(1+2 \alpha) t}
$$


Proof. Choose $\phi_{h}=\mathbf{u}_{h}$ in (3.3) and use (2.3) to obtain

$$
\frac{1}{2} \frac{d}{d t}\left\|\mathbf{u}_{h}\right\|^{2}+\mu\left\|\nabla \mathbf{u}_{h}\right\|^{2}+\rho\left\|\nabla \cdot \mathbf{u}_{h}\right\|^{2}+\int_{0}^{t} \beta(t-\tau) a\left(\mathbf{u}_{h}(\tau), \mathbf{u}_{h}\right) d \tau \leq\left(\mathbf{f}, \mathbf{u}_{h}\right)
$$

We multiply both side by $e^{2 \alpha t}$ and integrate with respect to time from 0 to $t$. Then, a use of the CauchySchwarz inequality with the Young's inequality yields

$$
\begin{array}{r}
e^{2 \alpha t}\left\|\mathbf{u}_{h}(t)\right\|^{2}+2 \int_{0}^{t} e^{2 \alpha s}\left(\mu\left\|\nabla \mathbf{u}_{h}(s)\right\|^{2}+\rho\left\|\nabla \cdot \mathbf{u}_{h}(s)\right\|^{2}\right) d s+2 \int_{0}^{t} e^{2 \alpha s} \int_{0}^{s} \beta(s-\tau) a\left(\mathbf{u}_{h}(\tau), \mathbf{u}_{h}(s)\right) d \tau d s \\
\leq\left\|\mathbf{u}_{h}(0)\right\|^{2}+\int_{0}^{t} e^{2 \alpha s}\|\mathbf{f}\|^{2} d s+(1+2 \alpha) \int_{0}^{t} e^{2 \alpha s}\left\|\mathbf{u}_{h}(s)\right\|^{2} d s
\end{array}
$$

The double integration term on the left hand side is positive due to Lemma 2.1, hence, we drop it. Then, we use the Gronwall's lemma to arrive at

$$
e^{2 \alpha t}\left\|\mathbf{u}_{h}(t)\right\|^{2}+2 \int_{0}^{t} e^{2 \alpha s}\left(\mu\left\|\nabla \mathbf{u}_{h}(s)\right\|^{2}+\rho\left\|\nabla \cdot \mathbf{u}_{h}(s)\right\|^{2}\right) d s \leq\left(\left\|\mathbf{u}_{0 h}\right\|^{2}+\frac{\|\mathbf{f}\|_{\infty}^{2}}{2 \alpha}\left(e^{2 \alpha t}-1\right)\right) e^{(1+2 \alpha) t}
$$

We multiply both side by $e^{-2 \alpha t}$ to complete the rest of the proof.

Lemma 3.1 helps us to prove the local existence of the solution of (3.3). Once we have the solution of (3.3), then using this we can easily prove the existence of the solutions of (3.2). The proof is quit similar to that of [34], hence we skip it. And uniqueness is obtained on the quotient space $L_{h} / N_{h}$, where

$$
N_{h}=\left\{q_{h} \in L_{h}:\left(q_{h}, \nabla \cdot \phi_{h}\right)=0 \text { for } \phi_{h} \in \mathbf{H}_{h}\right\} .
$$

The norm of $L_{h} / N_{h}$ is given by

$$
\left\|q_{h}\right\|_{L^{2} / N_{h}}=\inf _{\chi_{h} \in N_{h}}\left\|q_{h}+\chi_{h}\right\|
$$

Since $\mathbf{J}_{h}$ is finite dimensional, the problem (3.3) leads to a system of nonlinear integro-differential equations with a stabilization term. For continuous dependence of the discrete pressure $p_{h}(t) \in L_{h} / N_{h}$ on the discrete velocity $\mathbf{u}_{h}(t) \in \mathbf{J}_{h}$, we assume the following discrete inf-sup (LBB) condition:

(B2' $^{\prime}$ For every $q_{h} \in L_{h}$, there exists a non-trivial function $\phi_{h} \in \mathbf{H}_{h}$ such that

$$
\left|\left(q_{h}, \nabla \cdot \phi_{h}\right)\right| \geq C\left\|\nabla \phi_{h}\right\|\left\|q_{h}\right\|_{L^{2} / N_{h}},
$$

where the constant $C>0$ is independent of $h$.

Moreover, we also assume that the following approximation property holds true for $\mathbf{J}_{h}$.

(B2) For every $\mathbf{w} \in \mathbf{J}_{1} \cap \mathbf{H}^{k+1}$, there exists an approximation $r_{h} \mathbf{w} \in \mathbf{J}_{h}$ such that

$$
\left\|\mathbf{w}-r_{h} \mathbf{w}\right\|+h\left\|\nabla\left(\mathbf{w}-r_{h} \mathbf{w}\right)\right\| \leq C h^{j+1}\|\mathbf{w}\|_{j+1}, \quad 0 \leq j \leq k .
$$

We define $L^{2}$-projection $P_{h}: \mathbf{L}^{2} \rightarrow \mathbf{J}_{h}$ satisfy the following properties for $0 \leq j \leq k$ [23]

$$
\left\{\begin{array}{l}
\left\|\boldsymbol{\phi}-P_{h} \boldsymbol{\phi}\right\|+h\left\|\nabla P_{h} \boldsymbol{\phi}\right\| \leq C h^{j}\|\boldsymbol{\phi}\|_{j}, \quad \forall \boldsymbol{\phi} \in \mathbf{J}_{h} \\
\left\|\boldsymbol{\phi}-P_{h} \boldsymbol{\phi}\right\|+h\left\|\nabla\left(\boldsymbol{\phi}-P_{h} \boldsymbol{\phi}\right)\right\| \leq C h^{j+1}\|\boldsymbol{\phi}\|_{j+1}, \quad \forall \boldsymbol{\phi} \in \mathbf{J}_{1}(\Omega) \cap \mathbf{H}^{k+1}(\Omega) .
\end{array}\right.
$$

Let us also consider the Lagrange interpolant $I_{h} \mathbf{u} \in \mathbf{H}_{h}$ of a continuous function $\mathbf{u}$ satisfying the following bounds (see [8, Theorem 4.4.4])

$$
\left\|\mathbf{u}-I_{h} \mathbf{u}\right\|_{W^{m, p}(K)} \leq C h^{n-m}\|\mathbf{u}\|_{W^{n, p}(K)}, \quad 0 \leq m \leq n \leq k+1,
$$

where $n>\frac{2}{p}$ when $1<p \leq \infty$ and $n \geq 2$ when $p=1$.

We now define the discrete operator $\Delta_{h}: \mathbf{H}_{h} \rightarrow \mathbf{H}_{h}$ through the bilinear form $a(\cdot, \cdot)$ as

$$
a\left(\mathbf{v}_{h}, \phi_{h}\right)=\left(-\Delta_{h} \mathbf{v}_{h}, \phi_{h}\right), \quad \forall \mathbf{v}_{h}, \phi_{h} \in \mathbf{H}_{h} .
$$

And the Stokes operator $\tilde{\Delta}=P \Delta$ as $\tilde{\Delta}_{h}=P_{h} \Delta_{h}$. The restriction of $\tilde{\Delta}_{h}$ to $\mathbf{J}_{h}$ is invertible and its inverse is denoted as $\tilde{\Delta}_{h}^{-1}$. We recall the discrete Sobolev norms on $\mathbf{J}_{h}$ (see [23]): For $r \in \mathbb{R}$, we define

$$
\left\|\mathbf{v}_{h}\right\|_{r}:=\left\|\left(-\tilde{\Delta}_{h}\right)^{r / 2} \mathbf{v}\right\|, \quad \mathbf{v}_{h} \in \mathbf{J}_{h}
$$


We note that $\left\|\mathbf{v}_{h}\right\|_{0}=\left\|\mathbf{v}_{h}\right\|$ and $\left\|\mathbf{v}_{h}\right\|_{1}=\left\|\nabla \mathbf{v}_{h}\right\|$. Also the norm $\left\|\tilde{\Delta}_{h}(\cdot)\right\|$ is equivalent to the norm $\|\cdot\|_{2}$ in $\mathbf{J}_{h}$ with constant independent of $h$.

We present below the error analysis due to the space discretization (time remains continuous). Our analysis will be divided in two parts, based on the regularity of the given initial data. First, we consider sufficiently smooth initial data, that is, the initial velocity $\mathbf{u}_{0} \in \mathbf{J}_{1} \cap \mathbf{H}^{k}$, and then, we take smooth data, that is, $\mathbf{u}_{0} \in \mathbf{J}_{1} \cap \mathbf{H}^{2}$.

\subsection{Semidiscrete Error Estimate for Sufficiently Smooth Data}

In this section, we derive error bounds for the velocity and the pressure for the case in which regularity of the exact solution upto time $t=0$ is assumed, that is, the given data is as much regular as we need.

\subsubsection{Error bounds for velocity}

Since $\mathbf{J}_{h}$ is not a subspace of $\mathbf{J}_{1}$, the weak solution $\mathbf{u}$ satisfies

$$
\left(\mathbf{u}_{t}, \boldsymbol{\phi}_{h}\right)+\mu a\left(\mathbf{u}, \boldsymbol{\phi}_{h}\right)+b\left(\mathbf{u}, \mathbf{u}, \boldsymbol{\phi}_{h}\right)+\int_{0}^{t} \beta(t-s) a\left(\mathbf{u}(s), \boldsymbol{\phi}_{h}\right) d s=(\mathbf{f}, \boldsymbol{\phi})+\left(p, \nabla \cdot \boldsymbol{\phi}_{h}\right), \forall \boldsymbol{\phi}_{h} \in \mathbf{J}_{h} .
$$

Define $\mathbf{e}=\mathbf{u}-\mathbf{u}_{h}$, then subtract (3.3) from (3.5) and use $\nabla \cdot \mathbf{u}=0$ to obtain the following error equation

$$
\begin{array}{r}
\left(\mathbf{e}_{t}, \phi_{h}\right)+\mu a\left(\mathbf{e}, \phi_{h}\right)+\int_{0}^{t} \beta(t-s) a\left(\mathbf{e}(s), \phi_{h}\right) d s+\rho\left(\nabla \cdot \mathbf{e}, \nabla \cdot \phi_{h}\right)=\left(p, \nabla \cdot \phi_{h}\right) \\
+b\left(\mathbf{u}_{h}, \mathbf{u}_{h}, \phi_{h}\right)-b\left(\mathbf{u}, \mathbf{u}, \phi_{h}\right), \forall \phi_{h} \in \mathbf{J}_{h} .
\end{array}
$$

Theorem 3.1. Assume that (A1)-(A3), (B1) and (B2) hold. Let $\alpha>0$ be such that $\mu-\left(\frac{\gamma}{\delta-\alpha}\right)^{2}>0$. Then, there exists a positive constant $C$ that does not depend on the inverse power of $\mu$, such that the following bounds hold for $t \in[0, T], T>0$

$$
\|\mathbf{e}(t)\|^{2}+\beta_{1} e^{-2 \alpha t} \int_{0}^{t} e^{2 \alpha s}\|\nabla \mathbf{e}(s)\|^{2} d s+\rho e^{-2 \alpha t} \int_{0}^{t} e^{2 \alpha s}\|\nabla \cdot \mathbf{e}(s)\|^{2} d s \leq C h^{2 k} e^{L(t)},
$$

where, $\beta_{1}=\mu-\left(\frac{\gamma}{\delta-\alpha}\right)^{2}>0$, and

$$
L(t)=\int_{0}^{t}\left(2 \alpha+4\|\nabla \mathbf{u}(s)\|_{\infty}+\left(1+\frac{4}{\rho}\right)\|\mathbf{u}(s)\|_{2}^{2}\right) d s
$$

and $C$ depends on

$$
\|\mathbf{u}(t)\|_{k}^{2}+e^{-2 \alpha t} \int_{0}^{t} e^{2 \alpha s}\left((\mu+4 \rho+2)\|\mathbf{u}(s)\|_{k+1}^{2}+\frac{4}{\rho}\|p(s)\|_{k}^{2}\right) d s .
$$

Proof. Choose $\phi_{h}=P_{h} \mathbf{e}=\mathbf{e}-\left(\mathbf{u}-P_{h} \mathbf{u}\right)$ in (3.6) to arrive at

$$
\begin{aligned}
\frac{1}{2} \frac{d}{d t}\|\mathbf{e}\|^{2} & +\mu\|\nabla \mathbf{e}\|^{2}+\rho\|\nabla \cdot \mathbf{e}\|^{2}+\int_{0}^{t} \beta(t-\tau) a(\mathbf{e}(\tau), \mathbf{e}) d \tau=\left(\mathbf{e}_{t}, \mathbf{u}-P_{h} \mathbf{u}\right)+\mu a\left(\mathbf{e}, \mathbf{u}-P_{h} \mathbf{u}\right) \\
& +\rho\left(\nabla \cdot \mathbf{e}, \nabla \cdot\left(\mathbf{u}-P_{h} \mathbf{u}\right)\right)+\int_{0}^{t} \beta(t-\tau) a\left(\mathbf{e}(\tau), \mathbf{u}-P_{h} \mathbf{u}\right) d \tau+\left(p, \nabla \cdot P_{h} \mathbf{e}\right)-\Lambda\left(P_{h} \mathbf{e}\right),
\end{aligned}
$$

where

$$
\Lambda\left(\phi_{h}\right)=b\left(\mathbf{u}, \mathbf{u}, \phi_{h}\right)-b\left(\mathbf{u}_{h}, \mathbf{u}_{h}, \boldsymbol{\phi}_{h}\right)=b\left(\mathbf{u}, \mathbf{e}, \boldsymbol{\phi}_{h}\right)+b\left(\mathbf{e}, \mathbf{u}, \boldsymbol{\phi}_{h}\right)-b\left(\mathbf{e}, \mathbf{e}, \boldsymbol{\phi}_{h}\right) .
$$

Now, using the definition of $P_{h}$, we tackle the first term as

$$
\left(\mathbf{e}_{t}, \mathbf{u}-P_{h} \mathbf{u}\right)=\left(\mathbf{u}_{t}-P_{h} \mathbf{u}_{t}+P_{h} \mathbf{u}_{t}-\mathbf{u}_{h t}, \mathbf{u}-P_{h} \mathbf{u}\right)=\left(\mathbf{u}_{t}-P_{h} \mathbf{u}_{t}, \mathbf{u}-P_{h} \mathbf{u}\right)=\frac{1}{2} \frac{d}{d t}\left\|\mathbf{u}-P_{h} \mathbf{u}\right\|^{2} .
$$


An application of the Cauchy-Schwarz inequality and the Young's inequality with (3.4) leads to

$$
\mu\left|a\left(\mathbf{e}, \mathbf{u}-P_{h} \mathbf{u}\right)\right| \leq \mu\|\nabla \mathbf{e}\|\left\|\nabla\left(\mathbf{u}-P_{h} \mathbf{u}\right)\right\| \leq C \mu h^{k}\|\mathbf{u}\|_{k+1}\|\nabla \mathbf{e}\| \leq \frac{C \mu}{2} h^{2 k}\|\mathbf{u}\|_{k+1}^{2}+\frac{\mu}{2}\|\nabla \mathbf{e}\|^{2} .
$$

A use of the Cauchy-Schwarz inequality and the Young's inequality with (3.4) and $\|\nabla \cdot \phi\| \leq C\|\nabla \phi\|$ yield

$$
\rho\left|\left(\nabla \cdot \mathbf{e}, \nabla \cdot\left(\mathbf{u}-P_{h} \mathbf{u}\right)\right)\right| \leq C \rho\|\nabla \cdot \mathbf{e}\|\left\|\nabla\left(\mathbf{u}-P_{h} \mathbf{u}\right)\right\| \leq 2 C \rho h^{2 k}\|\mathbf{u}\|_{k+1}^{2}+\frac{\rho}{8}\|\nabla \cdot \mathbf{e}\|^{2} .
$$

And the integration term on the right hand side can be estimated as

$$
\begin{aligned}
\int_{0}^{t} \beta(t-\tau) a\left(\mathbf{e}(\tau), \mathbf{u}-P_{h} \mathbf{u}\right) d \tau & \leq\left(\int_{0}^{t} \beta(t-\tau)\|\nabla \mathbf{e}(\tau)\| d \tau\right)\left\|\nabla\left(\mathbf{u}-P_{h} \mathbf{u}\right)\right\| \\
& \leq C h^{k}\left(\int_{0}^{t} \beta(t-\tau)\|\nabla \mathbf{e}(\tau)\| d \tau\right)\|\mathbf{u}\|_{k+1} \\
& \leq \frac{C}{2} h^{2 k}\|\mathbf{u}\|_{k+1}^{2}+\frac{1}{2}\left(\int_{0}^{t} \beta(t-\tau)\|\nabla \mathbf{e}(\tau)\| d \tau\right)^{2} .
\end{aligned}
$$

A use of discrete incompressibility condition, that is, $\left(j_{h} p, \nabla \cdot P_{h} \mathbf{e}=0\right)$, with the Cauchy-Schwarz inequality and the approximation property (B1) yields

$$
\left|\left(p, \nabla \cdot P_{h} \mathbf{e}\right)\right|=\left|\left(p-j_{h} p, \nabla \cdot P_{h} \mathbf{e}\right)\right| \leq C h^{k}\|p\|_{k}\|\nabla \cdot \mathbf{e}\| \leq \frac{2 C}{\rho} h^{2 k}\|p\|_{k}^{2}+\frac{\rho}{8}\|\nabla \cdot \mathbf{e}\|^{2} .
$$

Using (2.3), we can rewrite the nonlinear terms as

$$
\left|\Lambda\left(P_{h} \mathbf{e}\right)\right|=\left|-b\left(\mathbf{u}, \mathbf{e}, \mathbf{u}-P_{h} \mathbf{u}\right)+b(\mathbf{e}, \mathbf{u}, \mathbf{e})-b\left(\mathbf{e}, \mathbf{u}, \mathbf{u}-P_{h} \mathbf{u}\right)+b\left(\mathbf{e}, \mathbf{e}, \mathbf{u}-P_{h} \mathbf{u}\right)\right|
$$

We use (2.2) and (2.1) with the Hölder's inequality, the Garliardo-Nirenberg inequality, the Agmon's inequality, the Young's inequality and (3.4) to bound the first and third term on right hand side of (3.15) as

$$
\begin{aligned}
\left|b\left(\mathbf{u}, \mathbf{e}, \mathbf{u}-P_{h} \mathbf{u}\right)\right| & =\left|b\left(\mathbf{u}, \mathbf{u}-P_{h} \mathbf{u}, \mathbf{e}\right)\right| \\
& =\left((\mathbf{u} \cdot \nabla)\left(\mathbf{u}-P_{h} \mathbf{u}\right), \mathbf{e}\right)+\frac{1}{2}\left((\nabla \cdot \mathbf{u})\left(\mathbf{u}-P_{h} \mathbf{u}\right), \mathbf{e}\right) \mid \\
& \leq\|\mathbf{u}\|_{\infty}\left\|\nabla\left(\mathbf{u}-P_{h} \mathbf{u}\right)\right\|\|\mathbf{e}\|+\frac{1}{2}\|\nabla \cdot \mathbf{u}\|_{L^{4}}\left\|\mathbf{u}-P_{h} \mathbf{u}\right\|_{L^{4}}\|\mathbf{e}\| \\
& \leq C h^{k}\|\mathbf{e}\|\|\mathbf{u}\|_{2}\|\mathbf{u}\|_{k+1} \\
& \leq \frac{C}{2} h^{2 k}\|\mathbf{u}\|_{k+1}^{2}+\frac{C}{2}\|\mathbf{u}\|_{2}^{2}\|\mathbf{e}\|^{2} .
\end{aligned}
$$

And

$$
\begin{aligned}
\left|b\left(\mathbf{e}, \mathbf{u}, \mathbf{u}-P_{h} \mathbf{u}\right)\right| & =\left|\frac{1}{2}\left((\mathbf{e} \cdot \nabla) \mathbf{u}, \mathbf{u}-P_{h} \mathbf{u}\right)-\frac{1}{2}\left((\mathbf{e} \cdot \nabla)\left(\mathbf{u}-P_{h} \mathbf{u}\right), \mathbf{u}\right)\right| \\
& \leq \frac{1}{2}\left(\|\mathbf{e}\|\|\nabla \mathbf{u}\|_{L^{4}}\left\|\mathbf{u}-P_{h} \mathbf{u}\right\|_{L^{4}}+\|\mathbf{e}\|\left\|\nabla\left(\mathbf{u}-P_{h} \mathbf{u}\right)\right\|\|\mathbf{u}\|_{\infty}\right) \\
& \leq C h^{k}\|\mathbf{e}\|\|\mathbf{u}\|_{2}\|\mathbf{u}\|_{k+1} \\
& \leq \frac{C}{2} h^{2 k}\|\mathbf{u}\|_{k+1}^{2}+\frac{C}{2}\|\mathbf{u}\|_{2}^{2}\|\mathbf{e}\|^{2} .
\end{aligned}
$$

To bound the second term on right hand side of (3.15), use (2.1) with the Cauchy-Schwarz inequality and the Agmon's inequality as

$$
\begin{aligned}
|b(\mathbf{e}, \mathbf{u}, \mathbf{e})| & \leq((\mathbf{e} \cdot \nabla) \mathbf{u}, \mathbf{e})+\frac{1}{2}((\nabla \cdot \mathbf{e}) \mathbf{u}, \mathbf{e}) \\
& \leq\|\nabla \mathbf{u}\|_{\infty}\|\mathbf{e}\|^{2}+\frac{1}{2}\|\nabla \cdot \mathbf{e}\|\|\mathbf{u}\|_{\infty}\|\mathbf{e}\| \\
& \leq C\left(\|\nabla \mathbf{u}\|_{\infty}+\frac{1}{\rho}\|\mathbf{u}\|_{2}^{2}\right)\|\mathbf{e}\|^{2}+\frac{\rho}{8}\|\nabla \cdot \mathbf{e}\|^{2}
\end{aligned}
$$


For the last term on right hand side of (3.15), use (2.2) then doing similar as (3.18) to obtain

$$
\begin{aligned}
\left|b\left(\mathbf{e}, \mathbf{e}, \mathbf{u}-P_{h} \mathbf{u}\right)\right| & =\left|b\left(\mathbf{e}, \mathbf{u}-P_{h} \mathbf{u}, \mathbf{e}\right)\right| \\
& \leq C\left(\left\|\nabla\left(\mathbf{u}-P_{h} \mathbf{u}\right)\right\|_{\infty}+\frac{1}{\rho}\left\|\mathbf{u}-P_{h} \mathbf{u}\right\|_{\infty}^{2}\right)\|\mathbf{e}\|^{2}+\frac{\rho}{8}\|\nabla \cdot \mathbf{e}\|^{2} \\
& \leq C\left(\|\nabla \mathbf{u}\|_{\infty}+\frac{1}{\rho}\|\mathbf{u}\|_{2}^{2}\right)\|\mathbf{e}\|^{2}+\frac{\rho}{8}\|\nabla \cdot \mathbf{e}\|^{2} .
\end{aligned}
$$

Inserting (3.10)-(3.19) in (3.9) and then multiplying both side by $e^{2 \alpha t}$, we arrive at

$$
\begin{aligned}
\frac{1}{2} \frac{d}{d t} e^{2 \alpha t}\|\mathbf{e}\|^{2} & +\frac{\mu}{2} e^{2 \alpha t}\|\nabla \mathbf{e}\|^{2}+\frac{\rho}{2} e^{2 \alpha t}\|\nabla \cdot \mathbf{e}\|^{2}+e^{2 \alpha t} \int_{0}^{t} \beta(t-\tau) a(\mathbf{e}(\tau), \mathbf{e}) d \tau \\
\leq & \frac{1}{2} \frac{d}{d t} e^{2 \alpha t}\left\|\mathbf{u}-P_{h} \mathbf{u}\right\|^{2}-\alpha e^{2 \alpha t}\left\|\mathbf{u}-P_{h} \mathbf{u}\right\|^{2}+C h^{2 k} e^{2 \alpha t}\left(\left(\frac{\mu}{2}+2 \rho+1\right)\|\mathbf{u}\|_{k+1}^{2}+\frac{2}{\rho}\|p\|_{k}^{2}\right) \\
& +e^{2 \alpha t}\left(2\|\nabla \mathbf{u}\|_{\infty}+\left(\frac{1}{2}+\frac{2}{\rho}\right)\|\mathbf{u}\|_{2}^{2}+\alpha\right)\|\mathbf{e}\|^{2}+\frac{1}{2} e^{2 \alpha t}\left(\int_{0}^{t} \beta(t-\tau)\|\nabla \mathbf{e}(\tau)\| d \tau\right)^{2} .
\end{aligned}
$$

First we drop the second term on the right hand side of (3.20) and then integrate with respect to time from 0 to $t$ and use $\|\mathbf{e}(0)\|=\left\|\mathbf{u}(0)-P_{h} \mathbf{u}(0)\right\|$ to obtain

$$
\begin{aligned}
& e^{2 \alpha t}\|\mathbf{e}(t)\|^{2}+\mu \int_{0}^{t} e^{2 \alpha s}\|\nabla \mathbf{e}(s)\|^{2} d s+\rho \int_{0}^{t} e^{2 \alpha s}\|\nabla \cdot \mathbf{e}(s)\|^{2} d s+2 \int_{0}^{t} e^{2 \alpha s} \int_{0}^{s} \beta(s-\tau) a(\mathbf{e}(\tau), \mathbf{e}(s)) d \tau d s \\
& \leq e^{2 \alpha t}\left\|\mathbf{u}(t)-P_{h} \mathbf{u}(t)\right\|^{2}+C h^{2 k} \int_{0}^{t} e^{2 \alpha s}\left((\mu+4 \rho+2)\|\mathbf{u}(s)\|_{k+1}^{2}+\frac{4}{\rho}\|p(s)\|_{k}^{2}\right) d s \\
& \quad+\int_{0}^{t} e^{2 \alpha s}\left(2 \alpha+4\|\nabla \mathbf{u}\|_{\infty}+\left(1+\frac{4}{\rho}\right)\|\mathbf{u}\|_{2}^{2}\right)\|\mathbf{e}(s)\|^{2} d s+\int_{0}^{t} e^{2 \alpha s}\left(\int_{0}^{s} \beta(s-\tau)\|\nabla \mathbf{e}(\tau)\| d \tau\right)^{2} d s
\end{aligned}
$$

From Lemma 2.1, the double integration term on left hand side is positive, so can drop it and the double integration term on right hand side can be bounded as similar as (4.2) of [34, page 761] as

$$
\int_{0}^{t} e^{2 \alpha s}\left(\int_{0}^{s} \beta(s-\tau)\|\nabla \mathbf{e}(\tau)\| d \tau\right)^{2} d s \leq\left(\frac{\gamma}{\delta-\alpha}\right)^{2} \int_{0}^{t} e^{2 \alpha s}\|\nabla \mathbf{e}(s)\|^{2} d s .
$$

Now, use (3.22) in (3.21) with $\beta_{1}=\mu-\left(\frac{\gamma}{\delta-\alpha}\right)^{2}>0$ and use the Gronwall's lemma to conclude

$$
\begin{aligned}
& e^{2 \alpha t}\|\mathbf{e}(t)\|^{2}+\beta_{1} \int_{0}^{t} e^{2 \alpha s}\|\nabla \mathbf{e}(s)\|^{2} d s+\rho \int_{0}^{t} e^{2 \alpha s}\|\nabla \cdot \mathbf{e}(s)\|^{2} d s \\
& \quad \leq C h^{2 k} e^{L(t)}\left[e^{2 \alpha t}\|\mathbf{u}(t)\|_{k}^{2}+\int_{0}^{t} e^{2 \alpha s}\left((\mu+4 \rho+2)\|\mathbf{u}(s)\|_{k+1}^{2}+\frac{4}{\rho}\|p(s)\|_{k}^{2}\right) d s\right] .
\end{aligned}
$$

Multiply both side by $e^{-2 \alpha t}$, which completes the rest of proof.

Remark 3.1. In Theorem 3.1, such a choice of $\alpha>0$ is possible under the assumption that $\mu>(\nu-\mu)^{2}$ and by choosing $\alpha<\delta\left(1-\frac{\nu-\mu}{\sqrt{\mu}}\right)$.

Remark 3.2. From the assumption (A3), it is clear that $L(t)$ defined on (3.7) is bounded by $C t$ and the quantity in (3.8) is also bounded by $C$, where $C$ does not depend on $\mu^{-1}$.

Remark 3.3. For stable mixed finite element spaces $\left(P_{k}, P_{k-1}\right), k>1$, the constant $C$ of Theorem 3.1 does not depends on the inverse power of $\mu$, but it depends on $\rho$ and $\rho^{-1}$. This justifies the standard choice of grad-div stabilization parameter to be $\rho \approx 1$ (as for NSEs, see [31, 33]). However we have numerically verified that it depends on the mesh size, the viscosity and the finite element spaces as well. (Detailed discussion for Stokes and NSEs can be found in [3, 25].)

And for a pair of equal degree inf-sup stable finite element spaces like MINI element $\left(P_{1} b, P_{1}\right)$, the constant $C$ depends on

$$
\|\mathbf{u}(t)\|_{1}^{2}+e^{-2 \alpha t} \int_{0}^{t} e^{2 \alpha s}\left((\mu+4 \rho+2)\|\mathbf{u}(s)\|_{2}^{2}+\frac{4 h^{2}}{\rho}\|p(s)\|_{2}^{2}\right) d s,
$$

Then we can choose $\rho \approx h^{2}$ or $h$, which give us the optimal result. In other words, we can choose the stabilization parameter $\rho$ in a range of $h^{2}$ to 1 . 


\subsubsection{Error bounds for pressure}

Theorem 3.2. Let us assume that the hypothesis of the Lemma 3.1 holds true. Additionally, we assume that $\mathbf{u}_{t} \in L^{2}\left(0, T ; \mathbf{H}^{k-1}\right)$, then there exists a positive constant $C$ independent of $\mu^{-1}$, such that, for all $t>0$,

$$
e^{-2 \alpha t} \int_{0}^{t} e^{2 \alpha s}\left\|\left(p-p_{h}\right)(s)\right\|_{L^{2} / N_{h}}^{2} d s \leq C h^{2 k} e^{L(t)},
$$

holds, where, $L(t)$ is defined in (3.7) and $C$ depends on the following

$$
\|\mathbf{u}(t)\|_{k}^{2}+e^{-2 \alpha t} \int_{0}^{t} e^{2 \alpha s}\left(\left\|\mathbf{u}_{s}(s)\right\|_{k-1}^{2}+(\mu+4 \rho+2)\|\mathbf{u}(s)\|_{k+1}^{2}+\frac{4}{\rho}\|p(s)\|_{k}^{2}\right) d s .
$$

To achieve a proof, we need some intermediate results. We start with splitting the pressure error $p-p_{h}$ as

$$
\left\|p-p_{h}\right\| \leq\left\|p-j_{h} p\right\|+\left\|j_{h} p-p_{h}\right\| .
$$

We just need to estimate the second term on the right hand side of (3.24) which we rewrite as

$$
\begin{aligned}
\left\|j_{h} p-p_{h}\right\|_{L^{2} / N_{h}} & \leq C \sup _{\boldsymbol{\phi}_{h} \in \mathbf{H}_{h} /\{0\}}\left\{\frac{\left|\left(j_{h} p-p_{h}, \nabla \cdot \boldsymbol{\phi}_{h}\right)\right|}{\left\|\nabla \boldsymbol{\phi}_{h}\right\|}\right\} \\
& \leq C\left(\left\|j_{h} p-p\right\|+\sup _{\boldsymbol{\phi}_{h} \in \mathbf{H}_{h} /\{0\}}\left\{\frac{\left|\left(p-p_{h}, \nabla \cdot \boldsymbol{\phi}_{h}\right)\right|}{\left\|\nabla \boldsymbol{\phi}_{h}\right\|}\right\}\right) .
\end{aligned}
$$

The first term on right hand side of (3.25) can be estimated by using (B2). And for the second term, we first look at the error equation in pressure obtained by subtracting (3.2) from (2.4):

$$
\left(p-p_{h}, \nabla \cdot \boldsymbol{\phi}_{h}\right)=\left(\mathbf{e}_{t}, \boldsymbol{\phi}_{h}\right)+\mu a\left(\mathbf{e}, \boldsymbol{\phi}_{h}\right)+\int_{0}^{t} \beta(t-s) a\left(\mathbf{e}(s), \boldsymbol{\phi}_{h}\right) d s+\rho\left(\nabla \cdot \mathbf{e}, \nabla \cdot \boldsymbol{\phi}_{h}\right)+\Lambda\left(\boldsymbol{\phi}_{h}\right),
$$

where

$$
\Lambda\left(\phi_{h}\right)=-b\left(\mathbf{u}, \mathbf{u}, \phi_{h}\right)+b\left(\mathbf{u}_{h}, \mathbf{u}_{h}, \phi_{h}\right)=-b\left(\mathbf{u}_{h}, \mathbf{e}, \phi_{h}\right)-b\left(\mathbf{e}, \mathbf{u}, \phi_{h}\right), \quad \phi_{h} \in \mathbf{H}_{h} .
$$

Similar to (3.16) and (3.17), we bound the nonlinear terms as

$$
\left|\Lambda\left(\phi_{h}\right)\right|=C\left(\|\mathbf{u}\|_{2}+\left\|\mathbf{u}_{h}\right\|_{2}\right)\|\mathbf{e}\|\|\nabla \phi\| .
$$

Since $\mathbf{u}$ is regular enough, $\mathbf{u}$ is continuous and hence, $\left\|I_{h} \mathbf{u}\right\|_{2} \leq C\|\mathbf{u}\|_{2}$, for some $C>0$. Then, using (3.1), (3.4) and Lemma 3.1, one can find

$$
\left\|\mathbf{u}_{h}\right\|_{2} \leq\left\|\mathbf{u}_{h}-I_{h} \mathbf{u}\right\|_{2}+\left\|I_{h} \mathbf{u}\right\|_{2} \leq C h^{-2}\left\|\mathbf{u}_{h}-I_{h} \mathbf{u}\right\|+C\|\mathbf{u}\|_{2} \leq C\|\mathbf{u}\|_{3} .
$$

Apply the Cauchy-Schwarz inequality and (3.27) in (3.26) to arrive at

$$
\left(p-p_{h}, \nabla \cdot \boldsymbol{\phi}_{h}\right) \leq C\left(\left\|\mathbf{e}_{t}\right\|_{-1 ; h}+\mu\|\nabla \mathbf{e}\|+\rho\|\nabla \cdot \mathbf{e}\|+\|\mathbf{u}\|_{3}\|\mathbf{e}\|+\int_{0}^{t} \beta(t-s)\|\nabla \mathbf{e}(s)\| d s\right)\left\|\nabla \boldsymbol{\phi}_{h}\right\|
$$

where,

$$
\left\|\mathbf{e}_{t}\right\|_{-1 ; h}=\sup \left\{\frac{<\mathbf{e}_{t}, \boldsymbol{\phi}_{h}>}{\left\|\nabla \boldsymbol{\phi}_{h}\right\|}: \boldsymbol{\phi}_{h} \in \mathbf{H}_{h}, \boldsymbol{\phi}_{h} \neq 0\right\}
$$

Since all the estimate on right hand side in (3.28) are known except $\left\|\mathbf{e}_{t}\right\|_{-1 ; h}$, we now derive $\left\|\mathbf{e}_{t}\right\|_{-1 ; h}$. As $\mathbf{H}_{h} \subset \mathbf{H}_{0}^{1}$, we note that

$$
\begin{aligned}
\left\|\mathbf{e}_{t}\right\|_{-1 ; h} & =\sup \left\{\frac{<\mathbf{e}_{t}, \boldsymbol{\phi}_{h}>}{\left\|\nabla \boldsymbol{\phi}_{h}\right\|}: \boldsymbol{\phi}_{h} \in \mathbf{H}_{h}, \boldsymbol{\phi}_{h} \neq 0\right\} \\
& \leq \sup \left\{\frac{<\mathbf{e}_{t}, \boldsymbol{\phi}>}{\|\nabla \boldsymbol{\phi}\|}: \boldsymbol{\phi} \in \mathbf{H}_{0}^{1}, \boldsymbol{\phi} \neq 0\right\}=\left\|\mathbf{e}_{t}\right\|_{-1} .
\end{aligned}
$$


Lemma 3.2. The error $\mathbf{e}=\mathbf{u}-\mathbf{u}_{h}$ satisfies for $0<t<T$

$$
\left\|\mathbf{e}_{t}\right\|_{-1} \leq C\left(h^{k}\left(\left\|\mathbf{u}_{t}\right\|_{k-1}+\|p\|_{k}\right)+\mu\|\nabla \mathbf{e}\|+\rho\|\nabla \cdot \mathbf{e}\|+\|\mathbf{u}\|_{3}\|\mathbf{e}\|+\int_{0}^{t} \beta(t-s)\|\nabla \mathbf{e}(s)\| d s\right) .
$$

Proof. For any $\boldsymbol{\psi} \in \mathbf{H}_{0}^{1}$, use the orthogonal projection $P_{h}: \mathbf{L}^{2} \rightarrow \mathbf{J}_{h}$, we obtain using (3.6) with $\phi_{h}=P_{h} \boldsymbol{\psi}$

$$
\begin{aligned}
\left(\mathbf{e}_{t}, \boldsymbol{\psi}\right)= & \left(\mathbf{e}_{t}, \boldsymbol{\psi}-P_{h} \boldsymbol{\psi}\right)+\left(\mathbf{e}_{t}, P_{h} \boldsymbol{\psi}\right) \\
= & \left(\mathbf{e}_{t}, \boldsymbol{\psi}-P_{h} \boldsymbol{\psi}\right)-\mu a\left(\mathbf{e}, P_{h} \boldsymbol{\psi}\right)-\int_{0}^{t} \beta(t-s) a\left(\mathbf{e}(s), P_{h} \boldsymbol{\psi}\right) d s+\left(p, \nabla \cdot P_{h} \boldsymbol{\psi}\right) \\
& -\rho\left(\nabla \cdot \mathbf{e}, \nabla \cdot P_{h} \boldsymbol{\psi}\right)-\Lambda\left(P_{h} \boldsymbol{\psi}\right) .
\end{aligned}
$$

Using approximation property of $P_{h}$, we find that

$$
\left(\mathbf{e}_{t}, \boldsymbol{\psi}-P_{h} \boldsymbol{\psi}\right)=\left(\mathbf{u}_{t}-P_{h} \mathbf{u}_{t}, \boldsymbol{\psi}-P_{h} \boldsymbol{\psi}\right) \leq C h^{k}\left\|\mathbf{u}_{t}\right\|_{k-1}\|\nabla \boldsymbol{\psi}\|
$$

Also, using discrete incompressibility condition and $H^{1}$-stability of $P_{h}$, we get

$$
\left(p, \nabla \cdot P_{h} \boldsymbol{\psi}\right) \leq\left(p-j_{h} p, \nabla \cdot P_{h} \boldsymbol{\psi}\right) \leq C h^{k}\|p\|_{k}\|\nabla \boldsymbol{\psi}\|
$$

Now, substitute (3.30)-(3.31) in (3.29) and use (3.27) with $\phi_{h}=P_{h} \boldsymbol{\psi}$ to obtain

$$
\left(\mathbf{e}_{t}, \boldsymbol{\psi}\right) \leq C\left(h^{k}\left(\left\|\mathbf{u}_{t}\right\|_{k-1}+\|p\|_{k}\right)+\mu\|\nabla \mathbf{e}\|+\rho\|\nabla \cdot \mathbf{e}\|+\|\mathbf{u}\|_{3}\|\mathbf{e}\|+\int_{0}^{t} \beta(t-s)\|\nabla \mathbf{e}(s)\| d s\right)\|\nabla \boldsymbol{\psi}\|
$$

and therefore,

$$
\begin{aligned}
\left\|\mathbf{e}_{t}\right\|_{-1} & \leq \sup \left\{\frac{\left\langle\mathbf{e}_{t}, \boldsymbol{\phi}>\right.}{\|\nabla \boldsymbol{\phi}\|}: \boldsymbol{\phi} \in \mathbf{H}_{0}^{1}, \boldsymbol{\phi} \neq 0\right\} \\
& \leq C\left(h^{k}\left(\left\|\mathbf{u}_{t}\right\|_{k-1}+\|p\|_{k}\right)+\mu\|\nabla \mathbf{e}\|+\rho\|\nabla \cdot \mathbf{e}\|+\|\mathbf{u}\|_{3}\|\mathbf{e}\|+\int_{0}^{t} \beta(t-s)\|\nabla \mathbf{e}(s)\| d s\right)
\end{aligned}
$$

which completes the proof.

Proof of the Theorem 3.2: From (3.24), (3.25), (3.28) and Lemma 3.2, we obtain

$$
\begin{aligned}
\left\|\left(p-p_{h}\right)\right\|_{L^{2} / N_{h}}^{2} \leq C & \left(h^{2 k}\left(\left\|\mathbf{u}_{t}\right\|_{k-1}^{2}+\|p\|_{k}^{2}\right)+\mu\|\nabla \mathbf{e}\|^{2}+\rho\|\nabla \cdot \mathbf{e}\|^{2}+\|\mathbf{u}\|_{3}^{2}\|\mathbf{e}\|^{2}\right. \\
& \left.+\left(\int_{0}^{t} \beta(t-s)\|\nabla \mathbf{e}(s)\| d s\right)^{2}\right)
\end{aligned}
$$

We multiply both side by $e^{2 \alpha t}$ and integrate with respect to time from 0 to $t$. Then, the resulting double integration term can be written as a single integration similar to (3.22) and we finally reach at

$$
\begin{aligned}
\int_{0}^{t} e^{2 \alpha s}\left\|\left(p-p_{h}\right)(s)\right\|_{L^{2} / N_{h}}^{2} d s \leq C & \left(h^{2 k} \int_{0}^{t} e^{2 \alpha s}\left(\left\|\mathbf{u}_{s}(s)\right\|_{k-1}^{2}+\|p(s)\|_{k}^{2}\right) d s+\beta_{1} \int_{0}^{t} e^{2 \alpha s}\|\nabla \mathbf{e}(s)\|^{2} d s\right. \\
& \left.+\rho \int_{0}^{t} e^{2 \alpha s}\|\nabla \cdot \mathbf{e}(s)\|^{2} d s+\|\mathbf{e}(t)\|_{L^{\infty}}^{2} \int_{0}^{t} e^{2 \alpha s}\|\mathbf{u}(s)\|_{3}^{2} d s\right) .
\end{aligned}
$$

We use Theorem 3.1 and multiply both side by $e^{-2 \alpha t}$ to complete the rest of the proof.

\subsection{Semidiscrete Error Estimate for Smooth Data}

As discussed in the introduction, the assumption of sufficiently smooth initial data is not realistic. And so we restrict the initial velocity $\mathbf{u}_{0}$ to be just smooth, that is, $\mathbf{u}_{0} \in \mathbf{J}_{1} \cap \mathbf{H}^{2}$. The analysis of this section takes into account the lack of regularity at $t=0$. 
Theorem 3.3. Assume that $(\boldsymbol{A} 1),(\boldsymbol{A} 2),\left(\boldsymbol{A} 3^{\prime}\right),(B 1)$ and $(\boldsymbol{B} 2)$ hold. Let $\alpha>0$ be such that $\mu-\left(\frac{\gamma}{\delta-\alpha}\right)^{2}>0$. Then, there exists a positive constant $C$ as defined on Theorem 3.1, such that the following bounds hold for $t \in[0, T], T>0$

$$
\|\mathbf{e}(t)\|^{2}+\beta_{1} e^{-2 \alpha t} \int_{0}^{t} e^{2 \alpha s}\|\nabla \mathbf{e}(s)\|^{2} d s+\rho e^{-2 \alpha t} \int_{0}^{t} e^{2 \alpha s}\|\nabla \cdot \mathbf{e}(s)\|^{2} d s \leq C h^{2 k} e^{L(t)}, \quad k \in\{1,2\}
$$

and

$$
e^{-2 \alpha t} \int_{0}^{t} e^{2 \alpha s}\left\|\left(p-p_{h}\right)(s)\right\|_{L^{2} / N_{h}}^{2} d s \leq C h^{2 k} e^{L(t)}, \quad k \in\{1,2\}
$$

where, $\beta_{1}$, and $L(t)$ are defined on Theorem 3.1.

We skip the proof since it follows the proofs of Theorems 3.1 and 3.2 .

Remark 3.4. Unlike in the case of sufficiently smooth data, where the estimates of Theorems 3.1 and 3.2 are valid for all $k \geq 1$, here, in the case of smooth only data, these estimates remain valid only for $k \in\{1,2\}$. That is, for $k \geq 3$, for higher order approximations of velocity and pressure, we do not obtain higher order rate of convergence, but is restricted to second order convergence for velocity and pressure, in case of smooth only data, and in case the estimates do not depend on inverse power of $\mu$.

In view of the above remark, we look into the case $k \geq 3$ for smooth initial data. We set $\phi_{h}=P_{h} \mathbf{e}=$ $\mathbf{e}-\left(\mathbf{u}-P_{h} \mathbf{u}\right)$ in (3.6) and following the steps (3.9)-(3.19), we obtain (3.20), that is,

$$
\begin{aligned}
\frac{1}{2} \frac{d}{d t} e^{2 \alpha t}\|\mathbf{e}\|^{2} & +\frac{\mu}{2} e^{2 \alpha t}\|\nabla \mathbf{e}\|^{2}+\frac{\rho}{2} e^{2 \alpha t}\|\nabla \cdot \mathbf{e}\|^{2}+e^{2 \alpha t} \int_{0}^{t} \beta(t-\tau) a(\mathbf{e}(\tau), \mathbf{e}) d \tau \\
\leq & \frac{1}{2} \frac{d}{d t} e^{2 \alpha t}\left\|\mathbf{u}-P_{h} \mathbf{u}\right\|^{2}-\alpha e^{2 \alpha t}\left\|\mathbf{u}-P_{h} \mathbf{u}\right\|^{2}+C h^{2 k} e^{2 \alpha t}\left(\left(\frac{\mu}{2}+2 \rho+1\right)\|\mathbf{u}\|_{k+1}^{2}+\frac{2}{\rho}\|p\|_{k}^{2}\right) \\
& +e^{2 \alpha t}\left(2\|\nabla \mathbf{u}\|_{\infty}+\left(\frac{1}{2}+\frac{2}{\rho}\right)\|\mathbf{u}\|_{2}^{2}+\alpha\right)\|\mathbf{e}\|^{2}+\frac{1}{2} e^{2 \alpha t}\left(\int_{0}^{t} \beta(t-\tau)\|\nabla \mathbf{e}(\tau)\| d \tau\right)^{2} .
\end{aligned}
$$

Here we can not integrate with respect to time directly, since the third term on the right hand side of (3.32) is no longer integrable near $t=0$ for $k \geq 3$. For example, from $\left(\mathbf{A} \mathbf{3}^{\prime}\right)$, and for $k=3$, we have

$$
\int_{0}^{t} e^{2 \alpha s} \tau(s)\left(\|\mathbf{u}\|_{4}^{2}+\|p\|_{3}^{2}\right) d s \leq C .
$$

Here the kernel $\tau(t)$ compensates for the singularity at $t=0$ of the higher order estimates of the solutions. Keeping this in mind we multiply $(3.32)$ by $\tau^{k-2}(t)$ and use the fact $\sigma_{t}^{k-2}(t) \leq 2 \alpha \sigma^{k-2}(t)+(k-2) \sigma^{k-3}(t)$, where $\sigma^{k}(t)=(\tau(t))^{k} e^{2 \alpha t}$. Then we integrate the resulting inequality over time from 0 to $t$ to obtain

$$
\begin{aligned}
\sigma^{k-2}(t)\|\mathbf{e}(t)\|^{2}+ & \int_{0}^{t} \sigma^{k-2}(s)\left(\mu\|\nabla \mathbf{e}\|^{2} d s+\rho\|\nabla \cdot \mathbf{e}\|^{2}\right) d s+2 \int_{0}^{t} \sigma^{k-2}(s) \int_{0}^{s} \beta(s-\tau) a(\mathbf{e}(\tau), \mathbf{e}(s)) d \tau d s \\
\leq & \sigma^{k-2}(t)\left\|\mathbf{u}(t)-P_{h} \mathbf{u}(t)\right\|^{2}-\int_{0}^{t}\left(2 \alpha \sigma^{k-2}(s)+(k-2) \sigma^{k-3}(s)\right)\left\|\mathbf{u}(t)-P_{h} \mathbf{u}(t)\right\|^{2} d s \\
& +(k-2) \int_{0}^{t} \sigma^{k-3}(s)\|\mathbf{e}(s)\|^{2} d s+C h^{2 k} \int_{0}^{t} \sigma^{k-2}(s)\left((\mu+4 \rho+2)\|\mathbf{u}(s)\|_{k+1}^{2}+\frac{4}{\rho}\|p(s)\|_{k}^{2}\right) d s \\
& +\int_{0}^{t} \sigma^{k-2}(s)\left(2 \alpha+4\|\nabla \mathbf{u}\|_{\infty}+\left(1+\frac{4}{\rho}\right)\|\mathbf{u}\|_{2}^{2}\right)\|\mathbf{e}\|^{2} d s \\
& +\int_{0}^{t} \sigma^{k-2}(s)\left(\int_{0}^{s} \beta(s-\tau)\|\nabla \mathbf{e}(\tau)\| d \tau\right)^{2} d s .
\end{aligned}
$$

However the bound for the third term on the right hand side of (3.33), that is,

$$
(k-2) \int_{0}^{t} \sigma^{k-3}(s)\|\mathbf{e}(s)\|^{2} d s
$$


is no longer independent of inverse power of $\mu$. To see this for the case $k=3$, we first split the error e in two parts, as $\mathbf{e}=\mathbf{u}-\mathbf{u}_{h}=\left(\mathbf{u}-\mathbf{v}_{h}\right)+\left(\mathbf{v}_{h}-\mathbf{u}_{h}\right)$, where $\mathbf{v}_{h}:[0, T) \rightarrow \mathbf{J}_{h}$ is the auxiliary function satisfying

$$
\left(\mathbf{u}_{t}-\mathbf{v}_{h t}, \phi_{h}\right)+\mu a\left(\mathbf{u}-\mathbf{v}_{h}, \phi_{h}\right)+\int_{0}^{t} \beta(t-s) a\left(\mathbf{u}(\tau)-\mathbf{v}_{h}(\tau), \phi_{h}\right) d \tau=0 .
$$

Let $\boldsymbol{\xi}=\mathbf{u}-\mathbf{v}_{h}$, the choose $\boldsymbol{\phi}_{h}=P_{h}\left(-\Delta_{h}\right)^{-1} \boldsymbol{\xi}=\left(-\Delta_{h}\right)^{-1} \boldsymbol{\xi}-\left(-\Delta_{h}\right)^{-1}\left(\mathbf{u}-P_{h} \mathbf{u}\right)$ in (3.35) to obtain

$$
\begin{array}{r}
\frac{1}{2} \frac{d}{d t}\|\boldsymbol{\xi}\|_{-1}^{2}+\mu\|\boldsymbol{\xi}\|^{2}+\int_{0}^{t} \beta(t-\tau)(\boldsymbol{\xi}(\tau), \boldsymbol{\xi}) d \tau=\left(\boldsymbol{\xi}_{t},\left(-\Delta_{h}\right)^{-1}\left(\mathbf{u}-P_{h} \mathbf{u}\right)\right)+\mu\left(\boldsymbol{\xi}, \mathbf{u}-P_{h} \mathbf{u}\right) \\
+\int_{0}^{t} \beta(t-\tau)\left(\boldsymbol{\xi}(\tau), \mathbf{u}-P_{h} \mathbf{u}\right) d \tau
\end{array}
$$

A use of the properties of $P_{h}$, the Cauchy-Schwarz inequality and the Young's inequality yields

$$
\begin{array}{r}
\frac{d}{d t}\|\boldsymbol{\xi}\|_{-1}^{2}+\mu\|\boldsymbol{\xi}\|^{2}+2 \int_{0}^{t} \beta(t-\tau)(\boldsymbol{\xi}(\tau), \boldsymbol{\xi}) d \tau \leq \frac{d}{d t}\left\|\mathbf{u}-P_{h} \mathbf{u}\right\|_{-1}^{2}+C(\mu+1) h^{6}\|\mathbf{u}\|_{3}^{2} \\
+\left(\int_{0}^{t} \beta(t-\tau)\|\boldsymbol{\xi}(\tau)\| d \tau\right)^{2}
\end{array}
$$

We multiply both side by $e^{2 \alpha t}$ and integrate with respect to time to arrive at

$$
\begin{array}{r}
e^{2 \alpha t}\|\boldsymbol{\xi}(t)\|_{-1}^{2}+\mu \int_{0}^{t} e^{2 \alpha s}\|\boldsymbol{\xi}(s)\|^{2} d s+2 \int_{0}^{t} e^{2 \alpha s} \int_{0}^{s} \beta(s-\tau)(\boldsymbol{\xi}(\tau), \boldsymbol{\xi}(s)) d \tau d s \leq 2 \alpha \int_{0}^{t} e^{2 \alpha s}\|\boldsymbol{\xi}(s)\|_{-1}^{2} d s \\
+e^{2 \alpha t}\left\|\mathbf{u}-P_{h} \mathbf{u}\right\|_{-1}^{2}+C(\mu+1) h^{6} \int_{0}^{t} e^{2 \alpha s}\|\mathbf{u}(s)\|_{3}^{2} d s+\int_{0}^{t} e^{2 \alpha s}\left(\int_{0}^{s} \beta(s-\tau)\|\boldsymbol{\xi}(\tau)\| d \tau\right)^{2} d s
\end{array}
$$

We drop the double integration term on the left hand side of (3.36) and as in (3.22) we bound the last term on the right hand side. Now for the first term on the right hand side, an application of the orthogonal property of $P_{h}$ and Cauchy-Schwarz's inequality leads to

$$
\begin{aligned}
\left\|\mathbf{u}-P_{h} \mathbf{u}\right\|_{-1}^{2} & =\left(\mathbf{u}-P_{h} \mathbf{u}+P_{h} \mathbf{u}-\mathbf{v}_{h},\left(-\Delta_{h}\right)^{-1}\left(\mathbf{u}-P_{h} \mathbf{u}\right)\right)+\left(\mathbf{v}_{h}-P_{h} \mathbf{u},\left(-\Delta_{h}\right)^{-1}\left(\mathbf{u}-P_{h} \mathbf{u}\right)\right) \\
& =\left(\boldsymbol{\xi},\left(-\Delta_{h}\right)^{-1}\left(\mathbf{u}-P_{h} \mathbf{u}\right)\right) \leq\|\boldsymbol{\xi}\|_{-1}\left\|\mathbf{u}-P_{h} \mathbf{u}\right\|_{-1} .
\end{aligned}
$$

Above we have used the fact that $\boldsymbol{\xi}=\mathbf{u}-\mathbf{v}_{h}$. On simplifying, we find

$$
\left\|\mathbf{u}-P_{h} \mathbf{u}\right\|_{-1} \leq\|\boldsymbol{\xi}\|_{-1} \text {. }
$$

And finally, a use of the Gronwall's lemma and (3.37) in (3.36) give

$$
\left(\mu-\frac{\gamma^{2}}{(\delta-\alpha)^{2}}\right) \int_{0}^{t} e^{2 \alpha s}\|\boldsymbol{\xi}(s)\|^{2} d s \leq C e^{2 \alpha t}(\mu+1) h^{6} \int_{0}^{t} e^{2 \alpha s}\|\mathbf{u}(s)\|_{3}^{2} d s .
$$

We recall from Theorem 3.1 that $\beta_{1}=\mu-\frac{\gamma^{2}}{(\delta-\alpha)^{2}}>0$. Now the resulting estimate will depend on the inverse power of $\mu$. Using similar arguments we can show that the estimate of (3.34) will depend on the inverse power of $\mu$ for $k>3$. And as a result, so will the estimate of (3.33).

In order to show that only second order convergence is possible, in case estimates are independent of inverse power of $\mu$, and in case $k \geq 3$, we obtain (3.32) as earlier. But now we restrict ourselves to lower order projection properties, that is, $\left\|\nabla\left(\mathbf{u}-P_{h} \mathbf{u}\right)\right\| \leq C h^{2}\|\mathbf{u}\|_{3}$ etc., which no longer demands a time weight $\tau(t)$. Following the lines of argument for (3.20), we can obtained the desired result.

\section{Backward Euler Method}

In this section, we consider the full discretization of the finite element approximation (3.2). We apply a backward Euler method for time discretization. Let $\left\{t_{n}\right\}_{n=0}^{N}$ be a uniform partition of the time interval $[0, T]$ and $t_{n}=n \Delta t$ with time step $\Delta t>0$. We approximate the time derivative term by

$$
\partial_{t} \phi^{n}=\left(\phi^{n}-\phi^{n-1}\right) / \Delta t
$$


where $\phi^{n}=\phi\left(t_{n}\right)$ a sequence in $\mathbf{J}_{h}$ which is defined on $[0, T]$. Since backward Euler method is of first order in time, so for integration term, we apply right rectangle rule as

$$
q_{r}^{n}(\boldsymbol{\phi})=\Delta t \sum_{j=0}^{n} \beta\left(t_{n}-t_{j}\right) \phi^{j} \approx \int_{0}^{t} \beta\left(t_{n}-s\right) \phi(s) d s .
$$

And it is observed that the right rectangle rule [35] is positive in the sense that

$$
\Delta t \sum_{i=1}^{n} q_{r}^{i}(\boldsymbol{\phi})=\Delta t \sum_{i=1}^{n}\left(\Delta t \sum_{j=0}^{i} \beta\left(t_{n}-t_{j}\right) \phi^{j}\right) \boldsymbol{\phi}^{i} \geq 0 .
$$

Now the backward Euler method applied in (3.2) is stated as below: Find $\mathbf{U}^{n} \in \mathbf{H}_{h}$ and $P^{n} \in L_{h}$ such that for $\mathbf{U}(0)=P_{h} \mathbf{u}_{0}$ and $t>0$

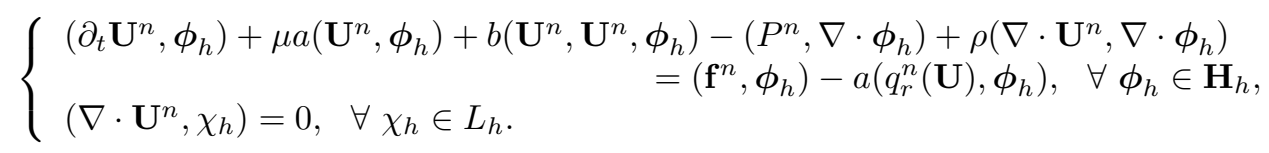

If we consider the discrete solution $\mathbf{U}^{n} \in \mathbf{J}_{h}$, then (4.2) becomes: Find $\mathbf{U}^{n} \in \mathbf{J}_{h}$ such that for $\mathbf{U}(0)=P_{h} \mathbf{u}_{0}$ and $t>0$

(4.3) $\left(\partial_{t} \mathbf{U}^{n}, \boldsymbol{\phi}_{h}\right)+\mu a\left(\mathbf{U}^{n}, \boldsymbol{\phi}_{h}\right)+b\left(\mathbf{U}^{n}, \mathbf{U}^{n}, \boldsymbol{\phi}_{h}\right)+\rho\left(\nabla \cdot \mathbf{U}^{n}, \nabla \cdot \boldsymbol{\phi}_{h}\right)=\left(\mathbf{f}^{n}, \boldsymbol{\phi}_{h}\right)-a\left(q_{r}^{n}(\mathbf{U}), \boldsymbol{\phi}_{h}\right), \quad \forall \boldsymbol{\phi}_{h} \in \mathbf{J}_{h}$,

Using variant of Brouwer fixed point theorem and standard uniqueness arguments, it is easy to show that the discrete problem (4.2) or (4.3) is well-posed. We can proof a priori bounds for the fully discrete solution $\left\{\mathbf{U}^{n}\right\}_{1 \leq n \leq N}$ similar to Lemma 3.1, which helps us to prove the local existence of the fully discrete solution (for a similar proof, see [5]).

\subsection{Fully Discrete Error Estimates for Sufficiently Smooth Data}

We define $\mathbf{u}\left(t_{n}\right)=\mathbf{u}^{n}, p\left(t_{n}\right)=p^{n}$ and set $\mathbf{e}^{n}=\mathbf{U}^{n}-\mathbf{u}^{n}$.

For the error equation, we consider (3.5) at $t=t_{n}$ and subtract the resulting equation from (4.3): For all $\phi_{h} \in \mathbf{J}_{h}$

$$
\begin{array}{r}
\left(\partial_{t} \mathbf{e}^{n}, \boldsymbol{\phi}_{h}\right)+\mu a\left(\mathbf{e}^{n}, \boldsymbol{\phi}_{h}\right)+\rho\left(\nabla \cdot \mathbf{e}^{n}, \nabla \cdot \boldsymbol{\phi}_{h}\right)+a\left(q_{r}^{n}(\mathbf{e}), \boldsymbol{\phi}_{h}\right) \\
=\left(p^{n}, \nabla \cdot \boldsymbol{\phi}_{h}\right)+R^{n}\left(\boldsymbol{\phi}_{h}\right)+\Lambda^{n}\left(\boldsymbol{\phi}_{h}\right)+E^{n}\left(\boldsymbol{\phi}_{h}\right),
\end{array}
$$

where

$$
\begin{aligned}
R^{n}\left(\boldsymbol{\phi}_{h}\right) & =\left(\mathbf{u}_{t}^{n}, \boldsymbol{\phi}_{h}\right)-\left(\partial_{t} \mathbf{u}^{n}, \boldsymbol{\phi}_{h}\right)=\left(\mathbf{u}_{t}^{n}, \boldsymbol{\phi}_{h}\right)-\frac{1}{\Delta t} \int_{t_{n-1}}^{t_{n}}\left(\mathbf{u}_{s}, \boldsymbol{\phi}_{h}\right) d s \\
& =\frac{1}{\Delta t} \int_{t_{n-1}}^{t_{n}}\left(s-t_{n-1}\right)\left(\mathbf{u}_{s s}, \boldsymbol{\phi}_{h}\right) d s \\
E^{n}\left(\boldsymbol{\phi}_{h}\right) & =\int_{0}^{t_{n}} \beta(t-s) a\left(\mathbf{u}(s), \boldsymbol{\phi}_{h}\right) d s-\Delta t \sum_{i=1}^{n} \beta\left(t_{n}-t_{i}\right) a\left(\mathbf{u}^{i}, \boldsymbol{\phi}_{h}\right) \\
& \leq C \sum_{i=1}^{n} \int_{t_{i-1}}^{t_{i}}\left(s-t_{i-1}\right)\left(\beta_{s}\left(t_{n}-s\right) a\left(\mathbf{u}(s), \boldsymbol{\phi}_{h}\right)+\beta\left(t_{n}-s\right) a\left(\mathbf{u}_{s}(s), \boldsymbol{\phi}_{h}\right)\right) d s . \\
\Lambda^{n}\left(\boldsymbol{\phi}_{h}\right) & =b\left(\mathbf{u}^{n}, \mathbf{u}^{n}, \boldsymbol{\phi}_{h}\right)-b\left(\mathbf{U}^{n}, \mathbf{U}^{n}, \boldsymbol{\phi}_{h}\right)=b\left(\mathbf{e}^{n}, \mathbf{e}^{n}, \boldsymbol{\phi}_{h}\right)-b\left(\mathbf{u}^{n}, \mathbf{e}^{n}, \boldsymbol{\phi}_{h}\right)-b\left(\mathbf{e}^{n}, \mathbf{u}^{n}, \boldsymbol{\phi}_{h}\right) .
\end{aligned}
$$

\subsubsection{Fully discrete error bounds for velocity}

In this section, we consider the exact solution to be sufficiently smooth. Our main result of this section is as follows: 
Theorem 4.1. Let the initial velocity satisfy $\mathbf{u}_{0} \in \mathbf{H}^{\max \{3, k\}}$ and let all other assumptions of Theorem 3.1 hold true. Further, let $\mathbf{u}_{t} \in L^{2}\left(0, T ; \mathbf{H}^{2}\right) \cap L^{2}\left(0, T ; \mathbf{H}^{k}\right)$ and $\mathbf{u}_{t t} \in L^{2}\left(0, T ; \mathbf{L}^{2}\right)$.

Then there exists a positive constant $C$, independent of the inverse power of $\mu$, such that the following bounds hold for $1 \leq n \leq N$

$$
\left\|\mathbf{e}^{n}\right\|^{2}+\beta_{1} \Delta t e^{-2 \alpha t_{n}} \sum_{i=1}^{n} e^{2 \alpha t_{i}}\left\|\nabla \mathbf{e}^{i}\right\|^{2}+\rho \Delta t e^{-2 \alpha t_{n}} \sum_{i=1}^{n} e^{2 \alpha t_{i}}\left\|\nabla \cdot \mathbf{e}^{i}\right\|^{2} \leq C e^{\hat{L}^{n}}\left(K_{1}\left(t_{n}\right) h^{2 k}+K_{2}\left(t_{n}\right)(\Delta t)^{2}\right),
$$

where $\beta_{1}=\mu-\left(\frac{\gamma}{\delta-\alpha}\right)^{2}>0$, and

$$
\begin{aligned}
\hat{L}^{n} & =\sum_{i=1}^{n}\left(C(\alpha)+4\left\|\nabla \mathbf{u}^{i}\right\|_{\infty}+\left(1+\frac{4}{\rho}\right)\left\|\mathbf{u}^{i}\right\|_{2}^{2}\right), \\
K_{1}\left(t_{n}\right) & =\left\|\mathbf{u}\left(t_{n}\right)\right\|_{k}^{2}+e^{-2 \alpha t_{n}} \int_{0}^{t_{n}} e^{2 \alpha s}\left(\left\|\mathbf{u}_{s}(s)\right\|_{k}^{2}+(\mu+4 \rho+2)\|\mathbf{u}(s)\|_{k+1}^{2}+\frac{4}{\rho}\|p(s)\|_{k}^{2}\right) d s, \\
K_{2}\left(t_{n}\right) & =e^{-2 \alpha t_{n}} \int_{0}^{t_{n}} e^{2 \alpha s}\left(\left\|\mathbf{u}_{s s}(s)\right\|^{2}+\|\mathbf{u}(s)\|_{2}^{2}+\left\|\mathbf{u}_{s}(s)\right\|_{2}^{2}\right) d s .
\end{aligned}
$$

Proof. We take $n=i$ and $\phi_{h}=P_{h} \mathbf{e}^{i}=\mathbf{e}^{i}-\left(\mathbf{u}^{i}-P_{h} \mathbf{u}^{i}\right)$ in (4.4) to arrive at

$$
\begin{aligned}
& \left(\partial_{t} \mathbf{e}^{i}, \mathbf{e}^{i}\right)+\mu a\left(\mathbf{e}^{i}, \mathbf{e}^{i}\right)+\rho\left(\nabla \cdot \mathbf{e}^{i}, \nabla \cdot \mathbf{e}^{i}\right)+a\left(q_{r}^{i}(\mathbf{e}), \mathbf{e}^{i}\right)=\left(\partial_{t} \mathbf{e}^{i}, \mathbf{u}^{i}-P_{h} \mathbf{u}^{i}\right)+\mu a\left(\mathbf{e}^{i}, \mathbf{u}^{i}-P_{h} \mathbf{u}^{i}\right) \\
& \quad+\rho\left(\nabla \cdot \mathbf{e}^{i}, \nabla \cdot\left(\mathbf{u}^{i}-P_{h} \mathbf{u}^{i}\right)\right)+a\left(q_{r}^{i}(\mathbf{e}), \mathbf{u}^{i}-P_{h} \mathbf{u}^{i}\right)+\left(p^{n}, \nabla \cdot P_{h} \mathbf{e}^{i}\right)+R_{h}^{i}\left(P_{h} \mathbf{e}^{i}\right)+\Lambda_{h}^{i}\left(P_{h} \mathbf{e}^{i}\right)+E_{h}^{i}\left(P_{h} \mathbf{e}^{i}\right) .
\end{aligned}
$$

We note that

$$
\left(\partial_{t} \phi^{i}, \phi^{i}\right)=\frac{1}{\Delta t}\left(\phi^{i}-\phi^{i-1}, \phi^{i}\right)=\frac{1}{2} \partial_{t}\left\|\phi^{i}\right\|^{2}+\frac{\Delta t}{2}\left\|\partial_{t} \phi^{i}\right\|^{2} \geq \frac{1}{2} \partial_{t}\left\|\phi^{i}\right\|^{2} .
$$

And a use of the property of $P_{h}$ yields

$$
\left(\partial_{t} \mathbf{e}^{i}, \mathbf{u}^{i}-P_{h} \mathbf{u}^{i}\right)=\left(\partial_{t}\left(\mathbf{u}^{i}-P_{h} \mathbf{u}^{i}\right), \mathbf{u}^{i}-P_{h} \mathbf{u}^{i}\right) \leq C h^{2 k}\left(\frac{1}{2} \partial_{t}\left\|\mathbf{u}^{i}\right\|_{k}^{2}+\frac{\Delta t}{2}\left\|\partial_{t} \mathbf{u}^{i}\right\|_{k}^{2}\right) .
$$

Now, we use the Cauchy-Schwarz inequality and the Young's inequality along with (3.4), (4.9) and (4.10) to obtain

$$
\begin{aligned}
\partial_{t}\left\|\mathbf{e}^{i}\right\|^{2}+ & \mu\left\|\nabla \mathbf{e}^{i}\right\|^{2}+\frac{3 \rho}{2}\left\|\nabla \cdot \mathbf{e}^{i}\right\|^{2}+2 a\left(q_{r}^{i}(\mathbf{e}), \mathbf{e}^{i}\right) \leq C h^{2 k}\left(\partial_{t}\left\|\mathbf{u}^{i}\right\|_{k}^{2}+\Delta t\left\|\partial_{t} \mathbf{u}^{i}\right\|_{k}^{2}\right. \\
& \left.+(\mu+4 \rho+1)\left\|\mathbf{u}^{i}\right\|_{k+1}^{2}+\frac{4}{\rho}\left\|p^{i}\right\|_{k}^{2}\right)+\left(q_{r}^{i}(\|\nabla \mathbf{e}\|)\right)^{2}+2 R_{h}^{i}\left(P_{h} \mathbf{e}^{i}\right)+2 \Lambda_{h}^{i}\left(P_{h} \mathbf{e}^{i}\right)+2 E_{h}^{i}\left(P_{h} \mathbf{e}^{i}\right) .
\end{aligned}
$$

We multiply both side by $\Delta t e^{2 \alpha t_{i}}$ then sum over $i=1$ to $n$ to find that,

$$
\begin{aligned}
\Delta t \sum_{i=1}^{n} e^{2 \alpha t_{i}} \partial_{t}\left\|\mathbf{e}^{i}\right\|^{2}+ & \mu \Delta t \sum_{i=1}^{n} e^{2 \alpha t_{i}}\left\|\nabla \mathbf{e}^{i}\right\|^{2}+\frac{3 \rho}{2} \Delta t \sum_{i=1}^{n} e^{2 \alpha t_{i}}\left\|\nabla \cdot \mathbf{e}^{i}\right\|^{2}+2 \Delta t \sum_{i=1}^{n} e^{2 \alpha t_{i}} a\left(q_{r}^{i}(\mathbf{e}), \mathbf{e}^{i}\right) \\
\leq & C h^{2 k} \Delta t \sum_{i=1}^{n} e^{2 \alpha t_{i}}\left(\partial_{t}\left\|\mathbf{u}^{i}\right\|_{k}^{2}+\Delta t\left\|\partial_{t} \mathbf{u}^{i}\right\|_{k}^{2}+(\mu+4 \rho+1)\left\|\mathbf{u}^{i}\right\|_{k+1}^{2}+\frac{4}{\rho}\left\|p^{i}\right\|_{k}^{2}\right) \\
& +\Delta t \sum_{i=1}^{n} e^{2 \alpha t_{i}}\left(q_{r}^{i}(\|\nabla \mathbf{e}\|)\right)^{2}+2 \Delta t \sum_{i=1}^{n} e^{2 \alpha t_{i}}\left(R^{i}\left(P_{h} \mathbf{e}^{i}\right)+\Lambda^{i}\left(P_{h} \mathbf{e}^{i}\right)+E^{i}\left(P_{h} \mathbf{e}^{i}\right)\right) .
\end{aligned}
$$

The positivity property (4.1) gives us

$$
\Delta t \sum_{i=1}^{n} e^{2 \alpha t_{i}} a\left(q_{r}^{i}(\mathbf{e}), \mathbf{e}^{i}\right)=\Delta t \sum_{i=1}^{n} e^{2 \alpha t_{i}} \Delta t \sum_{j=1}^{i} \beta\left(t_{i}-t_{j}\right)\left\|\nabla \mathbf{e}^{j}\right\|\left\|\nabla \mathbf{e}^{i}\right\| \geq 0 .
$$

Similar to (3.22), with a use of Cauchy-Schwarz inequality and the change of order of summation, we can write the second term on the right hand side of (4.11) as

$$
\Delta t \sum_{i=1}^{n} e^{2 \alpha t_{i}}\left(q_{r}^{i}(\|\nabla \mathbf{e}\|)\right)^{2}=\Delta t \sum_{i=1}^{n} e^{2 \alpha t_{i}} \Delta t \sum_{j=0}^{i} \beta\left(t_{n}-t_{j}\right)\left\|\nabla \mathbf{e}^{j}\right\|^{2} \leq\left(\frac{\gamma}{\delta-\alpha}\right)^{2} \Delta t \sum_{i=1}^{n} e^{2 \alpha t_{i}}\left\|\nabla \mathbf{e}^{i}\right\|^{2}
$$


Now use the fact that

$$
\Delta t \sum_{i=1}^{n} e^{2 \alpha t_{i}} \partial_{t}\left\|\mathbf{e}^{i}\right\|^{2}=\sum_{i=1}^{n} e^{2 \alpha t_{i}}\left(\left\|\mathbf{e}^{i}\right\|^{2}-\left\|\mathbf{e}^{i-1}\right\|^{2}\right)=e^{2 \alpha t_{n}}\left\|\mathbf{e}^{n}\right\|^{2}-\sum_{i=1}^{n-1} e^{2 \alpha t_{i}}\left(e^{2 \alpha \Delta t}-1\right)\left\|\mathbf{e}^{i}\right\|^{2} .
$$

in (4.11) and then use (4.12) and (4.13) with $\beta_{1}=\mu-\left(\frac{\gamma}{\delta-\alpha}\right)^{2}>0$ to arrive at

$$
\begin{aligned}
e^{2 \alpha t_{n}}\left\|\mathbf{e}^{n}\right\|^{2} & +\beta_{1} \Delta t \sum_{i=1}^{n} e^{2 \alpha t_{i}}\left\|\nabla \mathbf{e}^{i}\right\|^{2}+\frac{3 \rho}{2} \Delta t \sum_{i=1}^{n} e^{2 \alpha t_{i}}\left\|\nabla \cdot \mathbf{e}^{i}\right\|^{2} \leq \sum_{i=1}^{n-1} e^{2 \alpha t_{i}}\left(e^{2 \alpha \Delta t}-1\right)\left\|\mathbf{e}^{i}\right\|^{2} \\
& +C h^{2 k}\left[e^{2 \alpha t_{n}}\left\|\mathbf{u}^{n}\right\|_{k}^{2}-\left\|\mathbf{u}_{0}\right\|^{2}-\sum_{i=1}^{n-1} e^{2 \alpha t_{i}}\left(e^{2 \alpha \Delta t}-1\right)\left\|\mathbf{u}^{i}\right\|^{2}+(\Delta t)^{2} \sum_{i=1}^{n} e^{2 \alpha t_{i}}\left\|\partial_{t} \mathbf{u}^{i}\right\|_{k}^{2}\right. \\
(4.14) \quad & \left.+\Delta t \sum_{i=1}^{n} e^{2 \alpha t_{i}}\left((\mu+4 \rho+1)\left\|\mathbf{u}^{i}\right\|_{k+1}^{2}+\frac{4}{\rho}\left\|p^{i}\right\|_{k}^{2}\right)\right]+2 \Delta t \sum_{i=1}^{n} e^{2 \alpha t_{i}}\left(R^{i}\left(P_{h} \mathbf{e}^{i}\right)+\Lambda^{i}\left(P_{h} \mathbf{e}^{i}\right)+E^{i}\left(P_{h} \mathbf{e}^{i}\right)\right) .
\end{aligned}
$$

The second and third terms within the bracket on the right hand side are positive, so we drop them and the third and fourth terms can be written as

$$
(\Delta t)^{2} \sum_{i=1}^{n} e^{2 \alpha t_{i}}\left\|\partial_{t} \mathbf{u}^{i}\right\|_{k}^{2} \leq(\Delta t)^{2} \sum_{i=1}^{n} e^{2 \alpha t_{i}}\left(\frac{1}{\Delta t} \int_{t_{i-1}}^{t_{i}}\left\|\mathbf{u}_{s}(s)\right\|_{k} d s\right)^{2} \leq C \int_{0}^{t_{n}} e^{2 \alpha s}\left\|\mathbf{u}_{s}(s)\right\|_{k}^{2} d s .
$$

And

$$
\Delta t \sum_{i=1}^{n} e^{2 \alpha t_{i}}\left((\mu+4 \rho+1)\left\|\mathbf{u}^{i}\right\|_{k+1}^{2}+\frac{4}{\rho}\left\|p^{i}\right\|_{k}^{2}\right) \leq \int_{0}^{t_{n}} e^{2 \alpha s}\left((\mu+4 \rho+1)\|\mathbf{u}(s)\|_{k+1}^{2}+\frac{4}{\rho}\|p(s)\|_{k}^{2}\right) d s
$$

A use of the Cauchy-Schwarz inequality and the Young's inequality with $t_{i-1} \leq t, t \in\left[t_{i-1}, t_{i}\right]$ in $(4.5)$ yields

$$
\begin{aligned}
\Delta t \sum_{i=1}^{n} e^{2 \alpha t_{i}} R^{i}\left(P_{h} \mathbf{e}^{i}\right) & \leq \Delta t \sum_{i=1}^{n} e^{2 \alpha t_{i}} \frac{1}{\Delta t} \int_{t_{i-1}}^{t_{i}}\left(s-t_{i-1}\right)\left\|\mathbf{u}_{s s}(s)\right\| d s\left\|P_{h} \mathbf{e}^{i}\right\| \\
& \leq \Delta t \sum_{i=1}^{n} e^{2 \alpha t_{i}}\left(\frac{1}{\Delta t} \int_{t_{i-1}}^{t_{i}}\left(s-t_{i-1}\right)\left\|\mathbf{u}_{s s}(s)\right\| d s\right)^{2}+\frac{1}{4} \Delta t \sum_{i=1}^{n} e^{2 \alpha t_{i}}\left\|\mathbf{e}^{i}\right\|^{2} \\
& \leq \Delta t \sum_{i=1}^{n} e^{2 \alpha t_{i}} \frac{1}{(\Delta t)^{2}}\left(\int_{t_{i-1}}^{t_{i}}\left(s-t_{i-1}\right)^{2} d s\right)\left(\int_{t_{i-1}}^{t_{i}}\left\|\mathbf{u}_{s s}(s)\right\|^{2} d s\right)+\frac{1}{4} \Delta t \sum_{i=1}^{n} e^{2 \alpha t_{i}}\left\|\mathbf{e}^{i}\right\|^{2} \\
& \leq(\Delta t)^{2} \sum_{i=1}^{n} e^{2 \alpha t_{i}} \int_{t_{i-1}}^{t_{i}}\left\|\mathbf{u}_{s s}(s)\right\|^{2} d s+\frac{1}{4} \Delta t \sum_{i=1}^{n} e^{2 \alpha t_{i}}\left\|\mathbf{e}^{i}\right\|^{2} \\
& \leq C e^{2 \alpha \Delta t}(\Delta t)^{2} \int_{0}^{t_{n}} e^{2 \alpha s}\left\|\mathbf{u}_{s s}(s)\right\|^{2} d s+\frac{1}{4} \Delta t \sum_{i=1}^{n} e^{2 \alpha t_{i}}\left\|\mathbf{e}^{i}\right\|^{2} .
\end{aligned}
$$

Again from (4.6)

$$
\begin{aligned}
& \Delta t \sum_{i=1}^{n} e^{2 \alpha t_{i}}\left|E^{i}\left(P_{h} \mathbf{e}^{i}\right)\right| \\
& \quad \leq C \Delta t \sum_{i=1}^{n} e^{2 \alpha t_{i}}\left(\sum_{j=1}^{i} \int_{t_{j-1}}^{t_{j}}\left(s-t_{j-1}\right)\left|\left(\beta_{s}\left(t_{i}-s\right)\|\tilde{\Delta} \mathbf{u}(s)\|+\beta\left(t_{i}-s\right)\left\|\tilde{\Delta} \mathbf{u}_{s}(s)\right\|\right)\right| d s\right)\left\|P_{h} \mathbf{e}^{i}\right\| \\
& \quad \leq C \Delta t \sum_{i=1}^{n} e^{2 \alpha t_{i}}\left(\sum_{j=1}^{i} \int_{t_{j-1}}^{t_{j}}\left(s-t_{j-1}\right) \beta\left(t_{i}-s\right)\left(\delta\|\mathbf{u}(s)\|_{2}+\left\|\mathbf{u}_{s}(s)\right\|_{2}\right) d s\right)^{2}+\frac{1}{4} \Delta t \sum_{i=1}^{n} e^{2 \alpha t_{i}}\left\|\mathbf{e}^{i}\right\|^{2} \\
& \quad \leq C(\Delta t)^{2} \int_{0}^{t_{n}} e^{2 \alpha s}\left(\|\mathbf{u}(s)\|_{2}^{2}+\left\|\mathbf{u}_{s}(s)\right\|_{2}^{2}\right) d s+\frac{1}{4} \Delta t \sum_{i=1}^{n} e^{2 \alpha t_{i}}\left\|\mathbf{e}^{i}\right\|^{2}
\end{aligned}
$$


Also, similar to (3.15)-(3.19), we can bound the nonlinear terms of (4.7) as

$$
\begin{aligned}
\left|\Lambda^{i}\left(P_{h} \mathbf{e}^{i}\right)\right| & =\left|-b\left(\mathbf{u}^{i}, \mathbf{e}^{i}, \mathbf{u}^{i}-P_{h} \mathbf{u}^{i}\right)+b\left(\mathbf{e}^{i}, \mathbf{u}^{i}, \mathbf{e}^{i}\right)-b\left(\mathbf{e}^{i}, \mathbf{u}^{i}, \mathbf{u}^{i}-P_{h} \mathbf{u}^{i}\right)+b\left(\mathbf{e}^{i}, \mathbf{e}^{i}, \mathbf{u}^{i}-P_{h} \mathbf{u}^{i}\right)\right| \\
& \leq C h^{2 k}\left\|\mathbf{u}^{i}\right\|_{k+1}^{2}+\left(2\left\|\nabla \mathbf{u}^{i}\right\|_{\infty}+\left(\frac{1}{2}+\frac{2}{\rho}\right)\left\|\mathbf{u}^{i}\right\|_{2}^{2}\right)\left\|\mathbf{e}^{i}\right\|^{2}+\frac{\rho}{4}\left\|\nabla \cdot \mathbf{e}^{i}\right\|^{2} .
\end{aligned}
$$

Hence,

$$
\begin{aligned}
\Delta t \sum_{i=1}^{n} e^{2 \alpha t_{i}}\left|\Lambda^{i}\left(P_{h} \mathbf{e}^{i}\right)\right| \leq & C h^{2 k} \Delta t \sum_{i=1}^{n} e^{2 \alpha t_{i}}\left\|\mathbf{u}^{i}\right\|_{k+1}^{2}+C \Delta t \sum_{i=1}^{n} e^{2 \alpha t_{i}}\left(2\left\|\nabla \mathbf{u}^{i}\right\|_{\infty}+\left(1+\frac{1}{2 \rho}\right)\left\|\mathbf{u}^{i}\right\|_{2}^{2}\right)\left\|\mathbf{e}^{i}\right\|^{2} \\
& +\frac{\rho}{4} \Delta t \sum_{i=1}^{n} e^{2 \alpha t_{i}}\left\|\nabla \cdot \mathbf{e}^{i}\right\|^{2}
\end{aligned}
$$

Now, we use (4.15)-(4.19) in (4.14) to arrive at

$$
\begin{aligned}
e^{2 \alpha t_{n}}\left\|\mathbf{e}^{n}\right\|^{2}+ & \beta_{1} \Delta t \sum_{i=1}^{n} e^{2 \alpha t_{i}}\left\|\nabla \mathbf{e}^{i}\right\|^{2}+\rho \Delta t \sum_{i=1}^{n} e^{2 \alpha t_{i}}\left\|\nabla \cdot \mathbf{e}^{i}\right\|^{2} \\
\leq & C h^{2 k}\left[e^{2 \alpha t_{n}}\left\|\mathbf{u}\left(t_{n}\right)\right\|_{k}^{2}+\int_{0}^{t_{n}} e^{2 \alpha s}\left(\left\|\mathbf{u}_{s}(s)\right\|_{k}^{2}+(\mu+4 \rho+2)\|\mathbf{u}(s)\|_{k+1}^{2}+\frac{4}{\rho}\|p(s)\|_{k}^{2}\right) d s\right] \\
& +C(\Delta t)^{2} \int_{0}^{t_{n}} e^{2 \alpha s}\left(\left\|\mathbf{u}_{s s}\right\|^{2}+\|\mathbf{u}(s)\|_{2}^{2}+\left\|\mathbf{u}_{s}(s)\right\|_{2}^{2}\right) d s \\
& +\Delta t \sum_{i=1}^{n} e^{2 \alpha t_{i}}\left(\frac{e^{2 \alpha \Delta t}-1}{\Delta t}+4\left\|\nabla \mathbf{u}^{i}\right\|_{\infty}+\left(1+\frac{4}{\rho}\right)\left\|\mathbf{u}^{i}\right\|_{2}^{2}\right)\left\|\mathbf{e}^{i}\right\|^{2}
\end{aligned}
$$

Note that $e^{2 \alpha \Delta t}-1 \leq C(\alpha) \Delta t$. We now apply the discrete Gronwall's Lemma and then multiply the resulting equation by $e^{-2 \alpha t_{n}}$ to complete the rest of the proof.

Remark 4.1. From (A3), $\hat{L}^{n}$ defined in (4.8) is bounded by $C t_{n}$ and $K_{1}\left(t_{n}\right)$ and $K_{2}\left(t_{n}\right)$ defined in (3.8) and (3.23) respectively, both are bounded by $C$, where $C$ is not dependent of inverse power of $\mu$.

\subsubsection{Fully discrete error bounds for pressure}

To obtain the fully discrete pressure error estimate, first we consider (3.5) with $t=t_{n}$ and subtract (4.2) from the resulting equation to arrive at

$$
\begin{aligned}
\left(p^{n}-P^{n}, \nabla \cdot \boldsymbol{\phi}_{h}\right)= & \left(\partial_{t} \mathbf{e}^{n}, \boldsymbol{\phi}_{h}\right)+\mu a\left(\mathbf{e}^{n}, \boldsymbol{\phi}_{h}\right)+\rho\left(\nabla \cdot \mathbf{e}^{n}, \nabla \cdot \boldsymbol{\phi}_{h}\right)+a\left(q_{r}^{n}(\mathbf{e}), \boldsymbol{\phi}_{h}\right) \\
& +R^{n}\left(\boldsymbol{\phi}_{h}\right)+\Lambda^{n}\left(\boldsymbol{\phi}_{h}\right)+E^{n}\left(\boldsymbol{\phi}_{h}\right) .
\end{aligned}
$$

A use of (4.5), (4.6), (4.7) with the Cauchy-Schwarz inequality yields

$$
\begin{aligned}
\left(p^{n}-P^{n}, \nabla \cdot \boldsymbol{\phi}_{h}\right) \leq C & {\left[\left\|\partial_{t} \mathbf{e}^{n}\right\|_{-1}+\mu\left\|\nabla \mathbf{e}^{n}\right\|+\rho\left\|\nabla \cdot \mathbf{e}^{n}\right\|+\left\|q_{r}^{n}(\nabla \mathbf{e})\right\|+\frac{1}{\Delta t} \int_{t_{n-1}}^{t_{n}}\left(s-t_{n-1}\right)\left\|\mathbf{u}_{s s}(s)\right\|_{-1} d s\right.} \\
& +\sum_{i=1}^{n} \int_{t_{i-1}}^{t_{i}}\left(s-t_{i-1}\right)\left(\beta_{s}\left(t_{n}-s\right)\|\nabla \mathbf{u}(s)\|+\beta\left(t_{n}-s\right)\left\|\nabla \mathbf{u}_{s}(s)\right\|\right) d s \\
& \left.+\left(\left\|\mathbf{u}^{n}\right\|_{2}+\left\|\mathbf{U}^{n}\right\|_{2}\right)\left\|\mathbf{e}^{n}\right\|\right]\left\|\nabla \boldsymbol{\phi}_{h}\right\| .
\end{aligned}
$$

Arguing as in the proof of Lemma 3.2, we can bound the first term on the right hand side of (4.20) as

$$
\begin{aligned}
\left\|\partial_{t} \mathbf{e}^{n}\right\|_{-1} \leq & C\left[h^{2 k}\left\|\partial_{t} \mathbf{u}^{n}\right\|_{k-1}+\mu\left\|\nabla \mathbf{e}^{n}\right\|+\rho\left\|\nabla \cdot \mathbf{e}^{n}\right\|+\left\|q_{r}^{n}(\nabla \mathbf{e})\right\|+\frac{1}{\Delta t} \int_{t_{n-1}}^{t_{n}}\left(s-t_{n-1}\right)\left\|\mathbf{u}_{s s}(s)\right\|_{-1} d s\right. \\
& \left.+\sum_{i=1}^{n} \int_{t_{i-1}}^{t_{i}}\left(s-t_{i-1}\right)\left(\beta_{s}\left(t_{n}-s\right)\|\nabla \mathbf{u}(s)\|+\beta\left(t_{n}-s\right)\left\|\nabla \mathbf{u}_{s}(s)\right\|\right) d s+\left(\left\|\mathbf{u}^{n}\right\|_{2}+\left\|\mathbf{U}^{n}\right\|_{2}\right)\left\|\mathbf{e}^{n}\right\|\right] .
\end{aligned}
$$


Incorporate (4.21) in (4.20) and divide the resulting inequality by $\left\|\nabla \phi_{h}\right\|, \phi_{h} \neq 0$. Similar to (3.25), we then have

$$
\begin{aligned}
\left\|p^{n}-P^{n}\right\|_{L^{2} / N_{h}} \leq C & {\left[h^{k}\left(\left\|\partial_{t} \mathbf{u}^{n}\right\|_{k-1}+\left\|p^{n}\right\|_{k}\right)+\mu\left\|\nabla \mathbf{e}^{n}\right\|+\rho\left\|\nabla \cdot \mathbf{e}^{n}\right\|+\left\|q_{r}^{n}(\nabla \mathbf{e})\right\|\right.} \\
& +\frac{1}{\Delta t} \int_{t_{n-1}}^{t_{n}}\left(s-t_{n-1}\right)\left\|\mathbf{u}_{s s}(s)\right\|_{-1} d s+\left(\left\|\mathbf{u}^{n}\right\|_{2}+\left\|\mathbf{U}^{n}\right\|_{2}\right)\left\|\mathbf{e}^{n}\right\| \\
& \left.+\sum_{i=1}^{n} \int_{t_{i-1}}^{t_{i}}\left(s-t_{i-1}\right)\left(\beta_{s}\left(t_{n}-s\right)\|\nabla \mathbf{u}(s)\|+\beta\left(t_{n}-s\right)\left\|\nabla \mathbf{u}_{s}(s)\right\|\right) d s\right] .
\end{aligned}
$$

Squaring and multiplying both side by $\Delta t e^{2 \alpha t_{n}}$ with $n=i$ and taking sum from $i=1$ to $n$ to obtain

$$
\begin{aligned}
\Delta t \sum_{i=1}^{n}\left\|p^{i}-P^{i}\right\|_{L^{2} / N_{h}}^{2} \leq C \Delta t \sum_{i=1}^{n} & {\left[h^{2 k}\left(\left\|\partial_{t} \mathbf{u}^{i}\right\|_{k-1}^{2}+\left\|p^{i}\right\|_{k}^{2}\right)+\mu\left\|\nabla \mathbf{e}^{i}\right\|^{2}+\rho\left\|\nabla \cdot \mathbf{e}^{i}\right\|^{2}+\left\|q_{r}^{i}(\nabla \mathbf{e})\right\|^{2}\right.} \\
+ & \left(\frac{1}{\Delta t} \int_{t_{i-1}}^{t_{i}}\left(s-t_{i-1}\right)\left\|\mathbf{u}_{s s}(s)\right\|{ }_{-1} d s\right)^{2}+\left(\left\|\mathbf{u}^{i}\right\|_{2}^{2}+\left\|\mathbf{U}^{i}\right\|_{2}^{2}\right)\left\|\mathbf{e}^{i}\right\|^{2} \\
+ & \left.\left(\sum_{j=1}^{i} \int_{t_{j-1}}^{t_{j}}\left(s-t_{j-1}\right)\left(\beta_{s}\left(t_{i}-s\right)\|\nabla \mathbf{u}(s)\|+\beta\left(t_{i}-s\right)\left\|\nabla \mathbf{u}_{s}(s)\right\|\right) d s\right)^{2}\right] .
\end{aligned}
$$

A use of Theorem 4.1 and the Young's inequality leads to

$$
\begin{aligned}
\Delta t \sum_{i=1}^{n}\left\|p^{i}-P^{i}\right\|_{L^{2} / N_{h}}^{2} \leq & C e^{\hat{L} t_{n}} h^{2 k}\left(\left\|\mathbf{u}\left(t_{n}\right)\right\|_{k}^{2}+e^{-2 \alpha t_{n}} \int_{0}^{t_{n}} e^{2 \alpha s}\left(\left\|\mathbf{u}_{s}(s)\right\|_{k}^{2}\right.\right. \\
& \left.\left.+(\mu+4 \rho+2)\|\mathbf{u}(s)\|_{k+1}^{2}+\frac{4}{\rho}\|p(s)\|_{k}^{2}\right) d s\right) \\
& +C e^{\hat{L} t_{n}}(\Delta t)^{2} e^{-2 \alpha t_{n}} \int_{0}^{t_{n}} e^{2 \alpha s}\left(\left\|\mathbf{u}_{s s}(s)\right\|^{2}+\|\mathbf{u}(s)\|_{2}^{2}+\left\|\mathbf{u}_{s}(s)\right\|_{2}^{2}\right) d s
\end{aligned}
$$

Multiply both sides by $e^{-2 \alpha t}$. We summarize our result in the following Theorem.

Theorem 4.2. Under the assumption of theorem 4.1, the following holds true:

$$
\Delta t e^{-2 \alpha t_{n}} \sum_{i=0}^{n} e^{2 \alpha t_{i}}\left\|p^{n}-P^{n}\right\|_{L^{2} / N_{h}}^{2} \leq C e^{\hat{L}^{n}}\left(K_{1}\left(t_{n}\right) h^{2 k}+K_{2}\left(t_{n}\right)(\Delta t)^{2}\right) .
$$

\subsection{Fully Discrete Error Estimates for Smooth Data}

We now consider, the initial data $\mathbf{u}_{0} \in \mathbf{J}_{1} \cap \mathbf{H}^{2}$. Then, the following result holds.

Theorem 4.3. Let the assumptions of Theorem 3.3 hold true. Further, let $\tau(t) \mathbf{u}_{t} \in L^{2}\left(0, T ; \mathbf{H}^{2}\right)$ and $\tau(t) \mathbf{u}_{t t} \in L^{2}\left(0, T ; \mathbf{L}^{2}\right)$. Then there exists a positive constant $C$, independent of the inverse power of $\mu$, such that the following bounds hold for $1 \leq n \leq N$ and $k \in\{1,2\}$

$$
\left\|\mathbf{e}^{n}\right\|^{2}+\beta_{1} \Delta t e^{-2 \alpha t_{n}} \sum_{i=1}^{n} e^{2 \alpha t_{i}}\left\|\nabla \mathbf{e}^{i}\right\|^{2}+\rho \Delta t e^{-2 \alpha t_{n}} \sum_{i=1}^{n} e^{2 \alpha t_{i}}\left\|\nabla \cdot \mathbf{e}^{i}\right\|^{2} \leq C e^{\hat{L}^{n}}\left(K_{3}\left(t_{n}\right) h^{2 k}+K_{4}\left(t_{n}\right) \Delta t\right),
$$

and

$$
\Delta t e^{-2 \alpha t_{n}} \sum_{i=0}^{n} e^{2 \alpha t_{i}}\left\|p^{n}-P^{n}\right\|_{L^{2} / N_{h}}^{2} \leq C e^{\hat{L}^{n}}\left(K_{3}\left(t_{n}\right) h^{2 k}+K_{4}\left(t_{n}\right) \Delta t\right),
$$

where $\beta_{1}=\mu-\left(\frac{\gamma}{\delta-\alpha}\right)^{2}>0$, and $\hat{L}^{n}$ is defined in (4.8), and

$$
K_{3}\left(t_{n}\right)=\left\|\mathbf{u}\left(t_{n}\right)\right\|_{k}^{2}+e^{-2 \alpha t_{n}} \int_{0}^{t_{n}} e^{2 \alpha s} \tau^{k-1}(s)\left(\left\|\mathbf{u}_{s}(s)\right\|_{k}^{2}+(\mu+4 \rho+2)\|\mathbf{u}(s)\|_{k+1}^{2}+\frac{4}{\rho}\|p(s)\|_{k}^{2}\right) d s,
$$


and

$$
K_{4}\left(t_{n}\right)=e^{-2 \alpha t_{n}} \int_{0}^{t_{n}} e^{2 \alpha s} \tau(s)\left(\left\|\mathbf{u}_{s s}(s)\right\|^{2}+\|\mathbf{u}(s)\|_{2}^{2}+\left\|\mathbf{u}_{s}(s)\right\|_{2}^{2}\right) d s .
$$

Proof. The proof goes in the similar way of the proof of Theorem 4.1 except the estimates $(4.15),(4.17)$ and (4.18) since $\left\|\mathbf{u}_{t t}(t)\right\|$ and $\left\|\mathbf{u}_{t}(t)\right\|_{2}$ are not integrable at $t=0$, when $\mathbf{u}_{0} \in \mathbf{J}_{1} \cap \mathbf{H}^{2}$.

For $k=1$, (4.15) will go through as it is but for $k=2$, we modify it as follows, keeping in mind $\tau\left(t_{n}\right) \leq$ $\tau\left(t_{n-1}\right)+\Delta t \leq C \tau(t)$ for $t \in\left[t_{n-1}, t_{n}\right]$

$$
\begin{aligned}
(\Delta t)^{2} \sum_{i=1}^{n} e^{2 \alpha t_{i}}\left\|\partial_{t} \mathbf{u}^{i}\right\|_{2}^{2} & \leq(\Delta t)^{2} \sum_{i=1}^{n} e^{2 \alpha t_{i}}\left(\frac{1}{\Delta t} \int_{t_{i-1}}^{t_{i}}\left\|\mathbf{u}_{s}(s)\right\|_{2} d s\right)^{2} \\
& \leq e^{2 \alpha \Delta t} \sum_{i=1}^{n}\left(\int_{t_{i-1}}^{t_{i}} \frac{1}{\tau\left(t_{i}\right)} d s\right)\left(\int_{t_{i-1}}^{t_{i}} \tau\left(t_{i}\right) e^{2 \alpha t_{i-1}}\left\|\mathbf{u}_{s}(s)\right\|_{2}^{2} d s\right) \\
& \leq e^{2 \alpha \Delta t} \sum_{i=1}^{n}\left(\frac{\Delta t}{\tau\left(t_{i}\right)}\right)\left(\int_{t_{i-1}}^{t_{i}} \sigma(s)\left\|\mathbf{u}_{s}(s)\right\|_{2}^{2} d s\right)
\end{aligned}
$$

When $0<t_{i}<1$, we have $\tau\left(t_{i}\right)=t_{i}=i \Delta t$. Hence

$$
\sum_{i=1}^{n}\left(\frac{\Delta t}{\tau\left(t_{i}\right)}\right)\left(\int_{t_{i-1}}^{t_{i}} \sigma(s)\left\|\mathbf{u}_{s}(s)\right\|_{2}^{2} d s\right) \leq \sum_{i=1}^{n} \frac{1}{i}\left(\int_{t_{i-1}}^{t_{i}} \sigma(s)\left\|\mathbf{u}_{s}(s)\right\|_{2}^{2} d s\right) \leq \int_{0}^{t_{n}} \sigma(s)\left\|\mathbf{u}_{s}(s)\right\|_{2}^{2} d s .
$$

And when $t_{i} \geq 1$, we have $\tau\left(t_{i}\right)=1$ and then

$$
\sum_{i=1}^{n}\left(\frac{\Delta t}{\tau\left(t_{i}\right)}\right)\left(\int_{t_{i-1}}^{t_{i}} \sigma(s)\left\|\mathbf{u}_{s}(s)\right\|_{2}^{2} d s\right) \leq \Delta t \sum_{i=1}^{n}\left(\int_{t_{i-1}}^{t_{i}} \sigma(s)\left\|\mathbf{u}_{s}(s)\right\|_{2}^{2} d s\right) \leq \Delta t \int_{0}^{t_{n}} \sigma(s)\left\|\mathbf{u}_{s}(s)\right\|_{2}^{2} d s .
$$

Next, we modify (4.17) for both $k=1,2$, using the fact $t-t_{i-1} \leq \tau(t)$ for $t \in\left[t_{i-1}, t_{i}\right]$ as

$$
\begin{aligned}
\Delta t \sum_{i=1}^{n} e^{2 \alpha t_{i}} R^{i}\left(P_{h} \mathbf{e}^{i}\right) & \leq \Delta t \sum_{i=1}^{n} e^{2 \alpha t_{i}} \frac{1}{\Delta t} \int_{t_{i-1}}^{t_{i}}\left(s-t_{i-1}\right)\left\|\mathbf{u}_{s s}(s)\right\| d s\left\|P_{h} \mathbf{e}^{i}\right\| \\
& \leq \Delta t \sum_{i=1}^{n} e^{2 \alpha t_{i}}\left(\frac{1}{\Delta t} \int_{t_{i-1}}^{t_{i}}\left(s-t_{i-1}\right)\left\|\mathbf{u}_{s s}(s)\right\| d s\right)^{2}+\frac{1}{4} \Delta t \sum_{i=1}^{n} e^{2 \alpha t_{i}}\left\|\mathbf{e}^{i}\right\|^{2} \\
& \leq \frac{1}{\Delta t} \sum_{i=1}^{n} e^{2 \alpha t_{i}}\left(\int_{t_{i-1}}^{t_{i}}\left(s-t_{i-1}\right) d s\right)\left(\int_{t_{i-1}}^{t_{i}}\left(s-t_{i-1}\right)\left\|\mathbf{u}_{s s}(s)\right\|^{2} d s\right)+\frac{1}{4} \Delta t \sum_{i=1}^{n} e^{2 \alpha t_{i}}\left\|\mathbf{e}^{i}\right\|^{2} \\
& \leq \Delta t \sum_{i=1}^{n} e^{2 \alpha t_{i}} \int_{t_{i-1}}^{t_{i}} \tau(s)\left\|\mathbf{u}_{s s}(s)\right\|^{2} d s+\frac{1}{4} \Delta t \sum_{i=1}^{n} e^{2 \alpha t_{i}}\left\|\mathbf{e}^{i}\right\|^{2} \\
& \leq C \Delta t \int_{0}^{t_{n}} e^{2 \alpha s} \tau(s)\left\|\mathbf{u}_{s s}(s)\right\|^{2} d s+\frac{1}{4} \Delta t \sum_{i=1}^{n} e^{2 \alpha t_{i}}\left\|\mathbf{e}^{i}\right\|^{2} .
\end{aligned}
$$

Similarly we modify (4.18) and obtain

$$
\Delta t \sum_{i=1}^{n} e^{2 \alpha t_{i}}\left|E^{i}\left(P_{h} \mathbf{e}^{i}\right)\right| \leq C \Delta t \int_{0}^{t_{n}} e^{2 \alpha s} \tau(s)\left(\|\mathbf{u}(s)\|_{2}^{2}+\left\|\mathbf{u}_{s}(s)\right\|_{2}^{2}\right) d s+\frac{1}{4} \Delta t \sum_{i=1}^{n} e^{2 \alpha t_{i}}\left\|\mathbf{e}^{i}\right\|^{2} .
$$

We use these modified estimates in the proof of Theorem 4.1 to complete the velocity estimates. And now based on these modified estimates we can easily obtain the pressure estimate, similar to Theorem 4.2 which completes the rest of the proof.

Remark 4.2. Similar to semidiscrete case, here also we can not extend the analysis for $k \geq 3$ to obtain better convergence rate. 


\section{$5 \quad$ Numerical Experiments}

In this section, we present some numerical experiments that verify the results of previous section. First, we verify the order of convergence of the error estimates for velocity and pressure. For simplicity, we use examples with known solution. In all cases, computation are done in FreeFem $++[21]$.

\subsection{Known Analytic Solutions}

We consider the Oldroyd model of order one in the domain $\Omega=[0,1] \times[0,1]$ subject to the homogeneous Dirichlet boundary condition. We approximate the equation using the Mini-element $\left(P_{1} b, P_{1}\right)$ and the TaylorHood element $\left(P_{2}, P_{1}\right)$ over a union jack (criss-cross) triangulation of $\Omega$. The domain is partitioned into triangles with sizes $h=1 / 4,1 / 8,1 / 16,1 / 32$ and $1 / 64$, respectively. To verify the theoretical results, we consider the following examples:

Example 5.1. For the experiment we take the forcing term $f(x, t)$ such that the solution of the problem to be,

$$
\begin{aligned}
u_{1}(x, t) & =-e^{t}(\cos (2 \pi x) \sin (2 \pi y)-\sin (2 \pi y)), \\
u_{2}(x, t) & =e^{t}(\sin (2 \pi x) \cos (2 \pi y)-\sin (2 \pi x)), \\
p(x, t) & =2 \pi e^{t}(\cos (2 \pi y)-\cos (2 \pi x)) .
\end{aligned}
$$

First, we consider stable equal order finite element pair (MINI-element) and we discretize the domain with mesh sizes $h=\frac{1}{4}, \frac{1}{8}, \frac{1}{16}, \frac{1}{32}$ and $\frac{1}{64}$ in each coordinate directions. We take different values of $\mu=$ $1,10^{-2}, 10^{-4}, 10^{-6}$ and $10^{-8}$ for each values of $h$ with fixed $\Delta t=\mathcal{O}(h), \delta=0.1$ and $\gamma=0.1 \mu$. In Fig 1 , we present the velocity and the pressure errors in $\mathbf{L}^{2}$-norm. For this case, we set the grad-div parameter $\rho=h^{2}$ for optimal values (see, Remark 3.3).

Next, we consider the well-known Taylor-Hood element. In this case, we discritize the domain with mesh
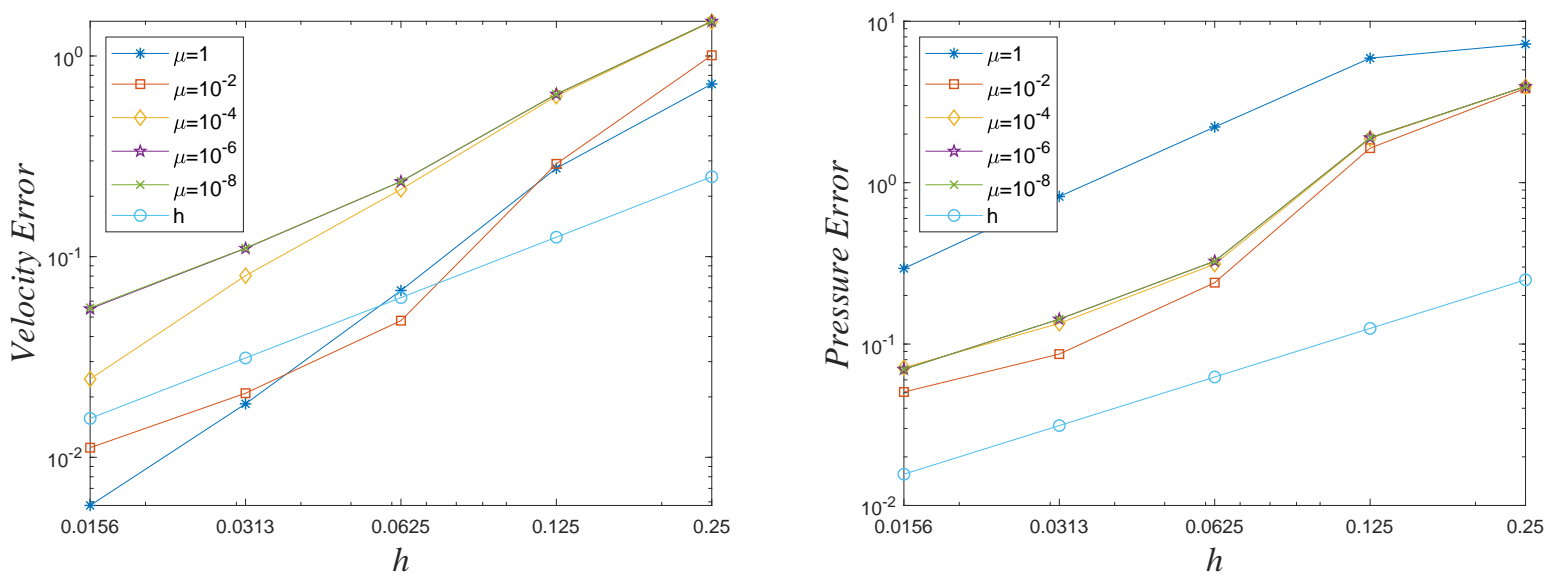

Figure 1: Velocity and pressure errors in $\mathbf{L}^{2}$-norm for Example 5.1 with $\rho=h^{2}$ for $\left(P_{1} b, P_{1}\right)$ element.

sizes $h=\frac{1}{4}, \frac{1}{8}, \frac{1}{16}, \frac{1}{32}$ and $\frac{1}{64}$. We present the velocity and the pressure errors for different values of $\mu$ as above with $\Delta t=\mathcal{O}\left(h^{2}\right), \delta=0.1$ and $\gamma=0.1 \mu$ in Fig 2. In this case, we set the grad-div parameter $\rho=0.25$ for optimal values. In Table 1, we present the errors and the rate of convergence for the velocity and the pressure in $L^{2}$-norm for $\mu=10^{-5}$.

We next look at a benchmark problem.

Example 5.2. In this example, we consider a benchmark problem related to a $2 D$ lid driven cavity flow on a unit square with zero body force. Also, no slip boundary condition is considered everywhere except the non zero velocity $\mathbf{u}=(1,0)^{T}$ on upper boundary.

For numerical experiments, we have chosen lines $(0.5, y)$ and $(x, 0.5)$. In Figure 3 , we present the comparison between velocity obtained by Ghia et. al. [18], velocity obtained without stabilization and velocity 



Figure 2: Velocity and pressure errors in $\mathbf{L}^{2}$-norm for Example 5.1 with $\rho=0.25$ for $\left(P_{2}, P_{1}\right)$ element.

\begin{tabular}{|c|c|c|c|c|c|c|c|c|}
\hline & \multicolumn{4}{|c|}{$P_{1} b-P_{1}$} & \multicolumn{4}{c|}{$P_{2}-P_{1}$} \\
\hline $\mathrm{h}$ & $\left\|u\left(t_{n}\right)-U^{n}\right\|$ & Rate & $\left\|p\left(t_{n}\right)-P^{n}\right\|$ & Rate & $\left\|u\left(t_{n}\right)-U^{n}\right\|$ & Rate & $\left\|p\left(t_{n}\right)-P^{n}\right\|$ & Rate \\
\hline $1 / 4$ & 1.48858661 & & 3.93977224 & & 0.63433473 & & 3.23651019 & \\
$1 / 8$ & 0.64373813 & 1.2094 & 1.89611585 & 1.0551 & 0.18140785 & 1.8060 & 0.86467803 & 1.9042 \\
$1 / 16$ & 0.23477703 & 1.4552 & 0.32472939 & 2.5457 & 0.04941585 & 1.8761 & 0.22448926 & 1.9455 \\
$1 / 32$ & 0.10624476 & 1.1439 & 0.14171058 & 1.1962 & 0.01246026 & 1.9874 & 0.05667220 & 1.9859 \\
$1 / 64$ & 0.04821498 & 1.1398 & 0.07036613 & 1.0100 & 0.00284780 & 2.1296 & 0.01429384 & 1.9872 \\
\hline
\end{tabular}

Table 1: Errors and convergence rates for $\nu=10^{-5}$ at time $t=1$.

obtained with stabilization of Oldroyd model of order one for final time $t=150$ for $\mu=10^{-4}$, with the choice of time step $k=0.01, \delta=0.1$ and $\gamma=0.1 \mu$. We also choose the stabilization parameter $\rho=0.1$. We approximate the equation using Taylor-Hood element over a union jack (criss-cross) triangulation of $\Omega$ and discretize the domain with mesh size $h=1 / 64$. From the graphs, it is observed that the velocity profiles with stabilization coincide with those of Ghia's results quite well in comparison with the velocity profile without stabilization for large time and for small $\nu$, (see Fig 3).
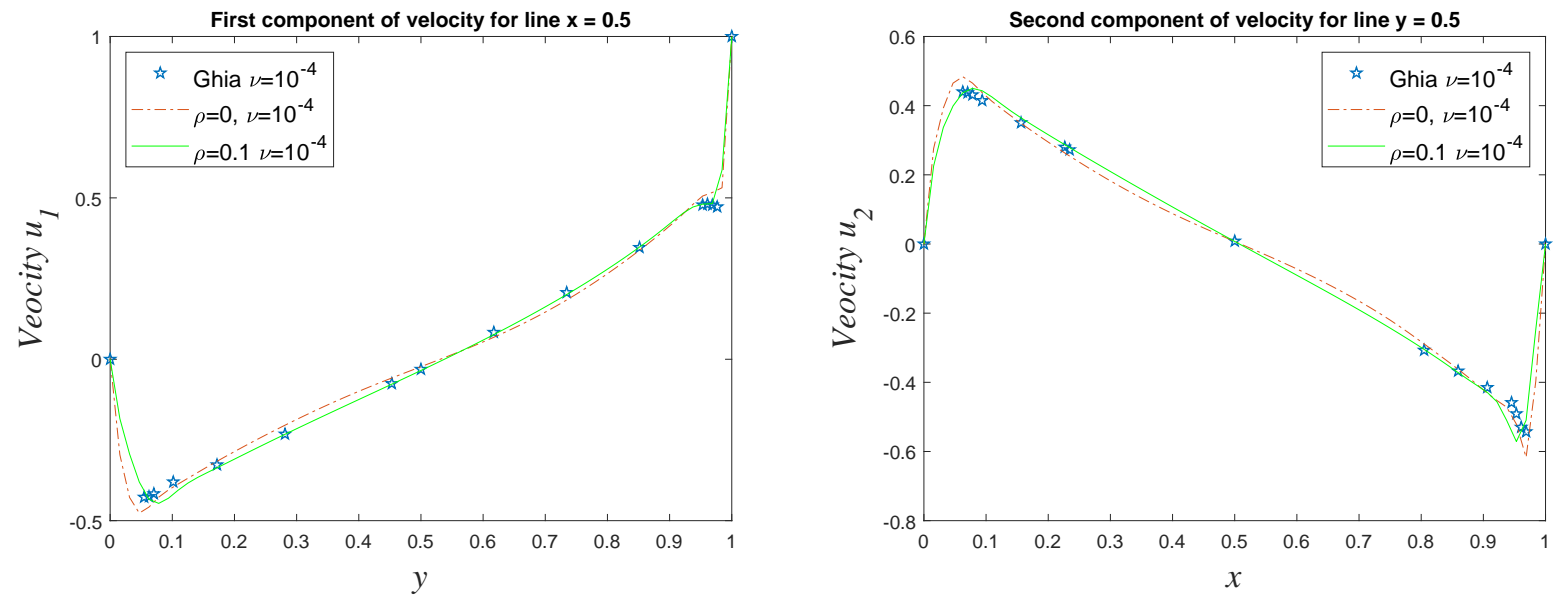

Figure 3: Velocity components for Example 5.2. 


\subsection{Choice of Grad-div parameter}

As discussed in the introduction, the choice of grad-div stabilization parameter plays a vital role in numerical simulations. Here we present a few numerical examples to find a suitable choice of grad-div parameter for the Oldroyd model of order one.

The numerical simulations are performed for different values of $\rho$ that lie between $10^{-3}$ to $10^{4}$, approximating the equation using the MINI element $\left(P_{1} b, P_{1}\right)$ and the Taylor-Hood element $\left(P_{2}, P_{1}\right)$. The numerical results are computed for three successively finer meshes with union jack (criss-cross) type triangulation with mesh sizes $h=1 / 8,1 / 16$ and $1 / 32$, and for $\mu=1,10^{-2}, 10^{-4}, 10^{-6}$ and $10^{-8}$. Figs 4 and 5 represent the velocity and the pressure errors graphs with respect to the grad-div stabilization parameter $\rho$ for the MINI element and the Taylor-Hood element, respectively. For different values of $\mu$, we mark the point where the error is minimum. Overall these figures give us a rough picture how the grad-div parameter $\rho$ changes with $h$ and $\mu$. We observe that for $\mathbf{L}^{2}$ error of velocity, a suitable range of $\rho$ would be from $10^{-1}$ to $10^{1}$. However, for $\mathbf{H}^{1}$ error of velocity and for $L^{2}$ error of pressure, a suitable range of $\rho$ is $10^{-1}$ to $10^{4}$.

We also present the values of grad-div parameter $\rho$, that minimize the $\mathbf{L}^{2}$ and $\mathbf{H}^{1}$ errors for the velocity and $L^{2}$ error for the pressure. We have used the known solution of Example 5.1. In the Tables 2 and 3, we present the corresponding minimum errors and the errors for standard choice of grad-div parameter $\rho=1$, for different values of $h$. We have used boldface for the minimizing value of $\rho$ in each case.
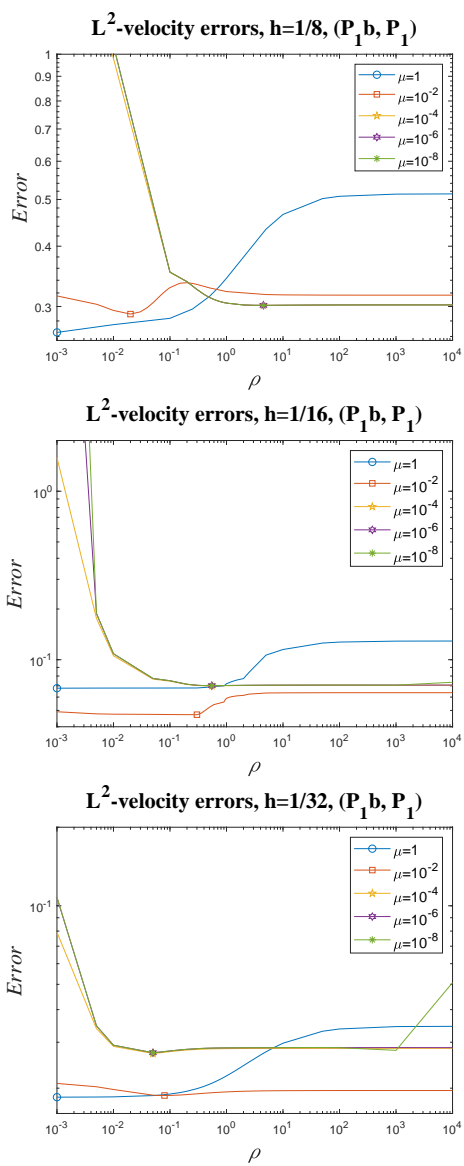



$H^{1}$-velocity errors, $h=1 / 16,\left(P_{1} b, P_{1}\right)$

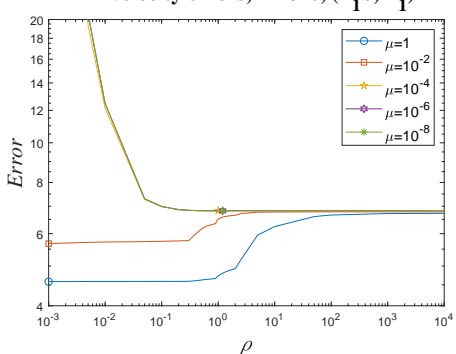

$\mathrm{H}^{\mathbf{1}}$-velocity errors, $\mathrm{h}=\mathbf{1} / \mathbf{3 2},\left(\mathbf{P}_{1} \mathrm{~b}, \mathrm{P}_{1}\right)$

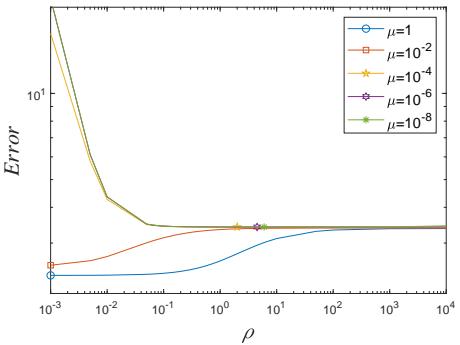

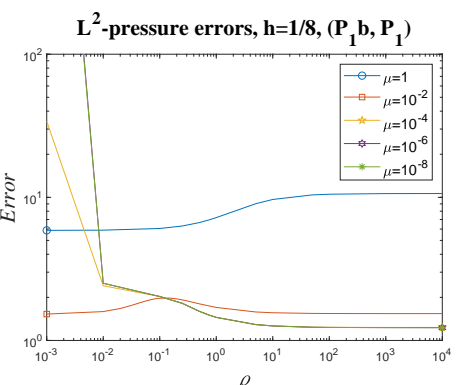
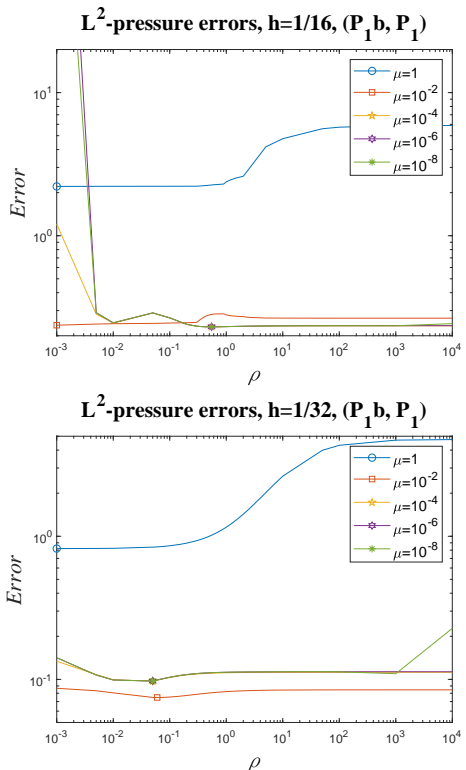

Figure 4: Velocity and pressure errors Vs stabilization parameter for $\left(P_{1} b, P_{1}\right)$ element.

\section{Conclusion}

We have considered here, an inf-sup mixed finite element method for Oldroyd model of order one with graddiv stabilization. We have obtained the error estimates in $L^{\infty}\left(\mathbf{L}^{2}\right)$-norm for the velocity and $L^{2}\left(\mathbf{L}^{2}\right)$-norm for the pressure in semidiscrete case as well as in the fully discrete case with the error bounds independent 

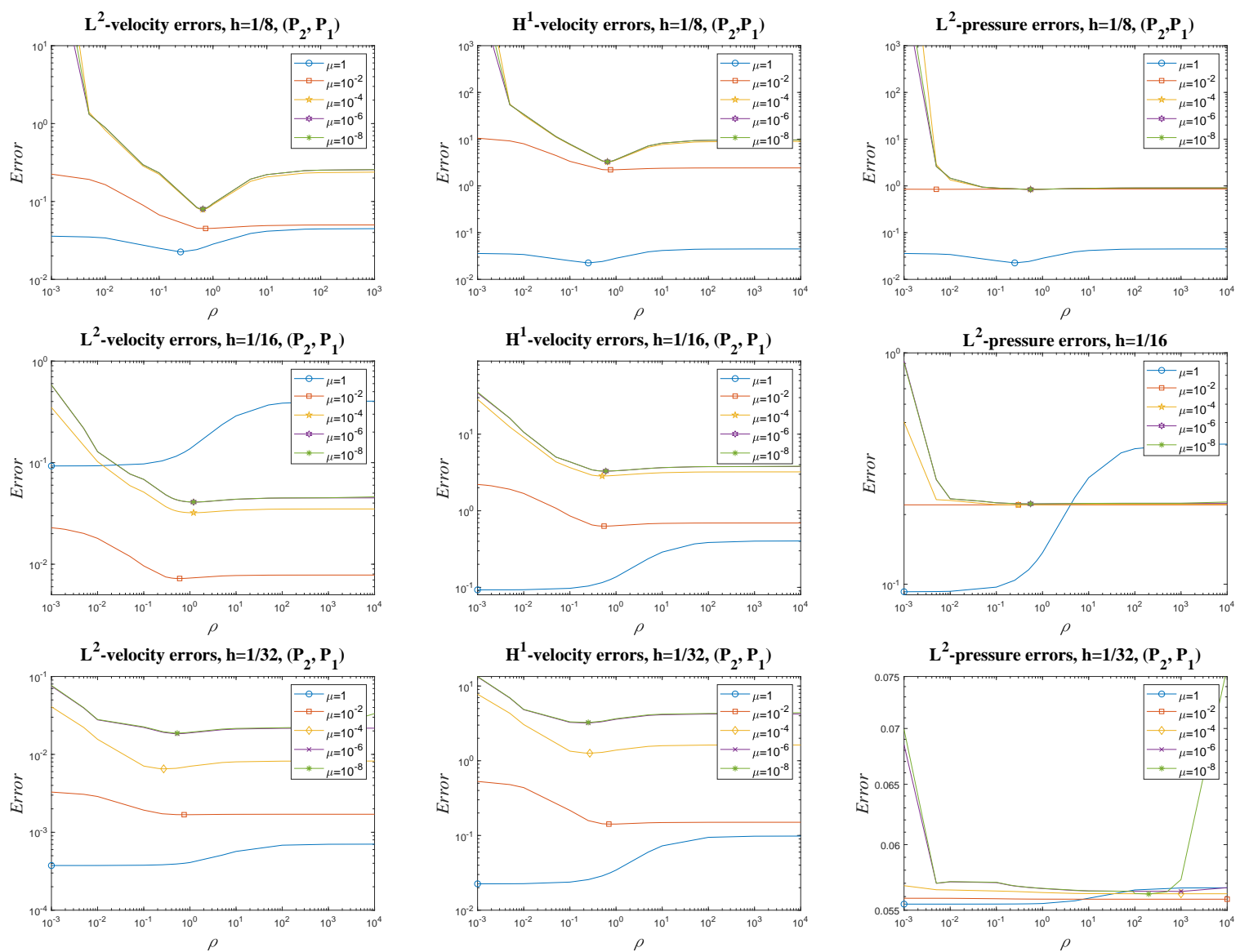

Figure 5: Velocity and pressure errors Vs stabilization parameter for $\left(P_{2}, P_{1}\right)$ element.

\begin{tabular}{|c|ccc|ccc|ccc|}
\hline & \multicolumn{2}{|c|}{ Velocity errors in $L^{2}$-norm } & \multicolumn{3}{|c|}{ Velocity errors in $H^{1}$-norm } & \multicolumn{3}{c|}{ Pressure errors in $L^{2}$-norm } \\
\hline$\mu$ & $\rho$ & Min & Std. $(\rho=1)$ & $\rho$ & Min & Std. $(\rho=1)$ & $\rho$ & Min & Std. $(\rho=1)$ \\
\hline $\mathrm{h}=1 / 8$ & & & & & & & & & \\
1 & $\mathbf{0 . 0 0 1}$ & 0.27419 & 0.34335 & $\mathbf{0 . 0 0 1}$ & 8.81884 & 10.05271 & $\mathbf{0 . 0 0 1}$ & 5.8799 & 7.21910 \\
$1 \mathrm{e}-2$ & $\mathbf{0 . 0 2}$ & 0.28931 & 0.32214 & $\mathbf{0 . 0 8}$ & 12.6806 & 13.31144 & $\mathbf{0 . 0 0 1}$ & 1.5288 & 1.70077 \\
$1 \mathrm{e}-4$ & $\mathbf{4 . 5}$ & 0.30166 & 0.30490 & $\mathbf{0 . 4}$ & 13.5764 & 13.60262 & $\mathbf{1 0 0 0 0}$ & 1.23236 & 1.45157 \\
$1 \mathrm{e}-6$ & $\mathbf{4 . 5}$ & 0.30166 & 0.30486 & $\mathbf{0 . 4}$ & 13.5823 & 13.60660 & $\mathbf{1 0 0 0 0}$ & 1.22929 & 1.44892 \\
$1 \mathrm{e}-8$ & $\mathbf{4 . 5}$ & 0.30166 & 0.30486 & $\mathbf{0 . 4}$ & 13.5823 & 13.60664 & $\mathbf{1 0 0 0 0}$ & 1.22746 & 1.44890 \\
$\mathrm{~h}=1 / 16$ & & & & & & & & & \\
1 & $\mathbf{0 . 0 0 1}$ & 0.06776 & 0.08406 & $\mathbf{0 . 0 0 1}$ & 4.58129 & 5.16855 & $\mathbf{0 . 0 0 1}$ & 2.21019 & 2.91551 \\
$1 \mathrm{e}-2$ & $\mathbf{0 . 0 1}$ & 0.04718 & 0.06249 & $\mathbf{0 . 0 0 1}$ & 5.67357 & 6.71433 & $\mathbf{0 . 0 0 1}$ & 0.23700 & 0.26881 \\
$1 \mathrm{e}-4$ & $\mathbf{0 . 5 5}$ & 0.07000 & 0.07011 & $\mathbf{1 . 0}$ & 6.82155 & 6.82155 & $\mathbf{0 . 5 5}$ & 0.23076 & 0.23147 \\
$1 \mathrm{e}-6$ & $\mathbf{0 . 5 5}$ & 0.07017 & 0.07030 & $\mathbf{1 . 2}$ & 6.82284 & 6.82290 & $\mathbf{0 . 5 5}$ & 0.23090 & 0.23174 \\
$1 \mathrm{e}-8$ & $\mathbf{0 . 5 5}$ & 0.07015 & 0.07030 & $\mathbf{1 . 2}$ & 6.82285 & 6.82291 & $\mathbf{0 . 5 5}$ & 0.23090 & 0.23175 \\
$\mathrm{~h}=1 / 32$ & & & & & & & & & \\
1 & $\mathbf{0 . 0 0 1}$ & 0.01848 & 0.02229 & $\mathbf{0 . 0 0 1}$ & 2.31312 & 2.59893 & $\mathbf{0 . 0 0 1}$ & 0.82050 & 1.15744 \\
$1 \mathrm{e}-2$ & $\mathbf{0 . 0 8}$ & 0.01875 & 0.01936 & $\mathbf{0 . 0 0 1}$ & 2.51021 & 3.34415 & $\mathbf{0 . 0 6}$ & 0.07475 & 0.08212 \\
$1 \mathrm{e}-4$ & $\mathbf{0 . 0 5}$ & 0.02717 & 0.02836 & $\mathbf{2 . 0}$ & 3.41308 & 3.41329 & $\mathbf{0 . 0 5}$ & 0.09716 & 0.11123 \\
$1 \mathrm{e}-6$ & $\mathbf{0 . 0 5}$ & 0.02735 & 0.02855 & $\mathbf{4 . 5}$ & 3.41332 & 3.41436 & $\mathbf{0 . 0 5}$ & 0.09740 & 0.11218 \\
$1 \mathrm{e}-8$ & $\mathbf{0 . 0 5}$ & 0.02735 & 0.02855 & $\mathbf{6 . 0}$ & 3.41334 & 3.41437 & $\mathbf{0 . 0 5}$ & 0.09740 & 0.11217 \\
\hline
\end{tabular}

Table 2: Minimum errors and corresponding stabilization parameter $\rho$ for $\left(P_{1} b, P_{1}\right)$ element

of the inverse power of $\mu$. We have carried out our analysis for both sufficiently smooth and smooth initial data. And finally we have briefly looked at suitable values of the grad-div parameter. 


\begin{tabular}{|c|ccc|ccc|ccc|}
\hline & \multicolumn{2}{|c|}{ Velocity errors in $L^{2}$-norm } & \multicolumn{3}{|c|}{ Velocity errors in $H^{1}$-norm } & \multicolumn{3}{|c|}{ Pressure errors in $L^{2}$-norm } \\
\hline$\mu$ & $\rho$ & Min & Std. $(\rho=1)$ & $\rho$ & Min & Std. $(\rho=1)$ & $\rho$ & Min & Std. $(\rho=1)$ \\
\hline $\mathrm{h}=1 / 8$ & & & & & & & & & \\
1 & $\mathbf{0 . 0 0 1}$ & 0.01011 & 0.01365 & $\mathbf{0 . 0 0 1}$ & 0.40921 & 0.54307 & $\mathbf{0 . 0 0 1}$ & 0.86810 & 0.88401 \\
$1 \mathrm{e}-2$ & $\mathbf{0 . 7 3}$ & 0.04502 & 0.04525 & $\mathbf{0 . 7 6}$ & 2.21141 & 2.21968 & $\mathbf{0 . 0 0 5}$ & 0.84583 & 0.85761 \\
$1 \mathrm{e}-4$ & $\mathbf{0 . 6 5}$ & 0.07898 & 0.09129 & $\mathbf{0 . 6 5}$ & 3.26339 & 3.57391 & $\mathbf{0 . 5 5}$ & 0.84359 & 0.84799 \\
$1 \mathrm{e}-6$ & $\mathbf{0 . 6 5}$ & 0.08016 & 0.09427 & $\mathbf{0 . 6 5}$ & 3.29902 & 3.66404 & $\mathbf{0 . 5 5}$ & 0.84148 & 0.84708 \\
$\mathrm{le}-8$ & $\mathbf{0 . 6 5}$ & 0.08017 & 0.09430 & $\mathbf{0 . 6 5}$ & 3.29939 & 3.66500 & $\mathbf{0 . 5 5}$ & 0.84146 & 0.84707 \\
$\mathrm{~h}=1 / 16$ & & & & & & & & & \\
1 & $\mathbf{0 . 0 0 1}$ & 0.00133 & 0.00182 & $\mathbf{0 . 0 0 1}$ & 0.09276 & 0.13683 & $\mathbf{0 . 0 0 1}$ & 0.22091 & 0.22190 \\
$1 \mathrm{e}-2$ & $\mathbf{0 . 6}$ & 0.00723 & 0.00731 & $\mathbf{0 . 5 5}$ & 0.63038 & 0.63938 & $\mathbf{0 . 3}$ & 0.22028 & 0.22029 \\
$\mathrm{l}-4$ & $\mathbf{1 . 2}$ & 0.03211 & 0.03214 & $\mathbf{0 . 5}$ & 2.84648 & 2.89919 & $\mathbf{0 . 3}$ & 0.22017 & 0.22060 \\
$1 \mathrm{e}-6$ & $\mathbf{1 . 2}$ & 0.04087 & 0.04098 & $\mathbf{0 . 6}$ & 3.29495 & 3.32761 & $\mathbf{0 . 5 5}$ & 0.22258 & 0.22268 \\
$1 \mathrm{e}-8$ & $\mathbf{1 . 2}$ & 0.04099 & 0.04110 & $\mathbf{0 . 6}$ & 3.30041 & 3.33304 & $\mathbf{0 . 5 5}$ & 0.22262 & 0.22271 \\
$\mathrm{~h}=1 / 32$ & & & & & & & & & \\
1 & $\mathbf{0 . 0 0 1}$ & 0.00037 & 0.00041 & $\mathbf{0 . 0 0 1}$ & 0.02253 & 0.03440 & $\mathbf{0 . 0 0 1}$ & 0.05545 & 0.05549 \\
$1 \mathrm{e}-2$ & $\mathbf{0 . 7 3}$ & 0.00168 & 0.00168 & $\mathbf{0 . 7}$ & 0.14200 & 0.14244 & $\mathbf{1 0 0 0 0}$ & 0.05581 & 0.05581 \\
$1 \mathrm{e}-4$ & $\mathbf{0 . 2 7}$ & 0.00652 & 0.00704 & $\mathbf{0 . 2 7}$ & 1.26200 & 1.38753 & $\mathbf{1 0 0 0}$ & 0.05621 & 0.05630 \\
$\mathrm{le}-6$ & $\mathbf{0 . 5 5}$ & 0.01876 & 0.01888 & $\mathbf{0 . 2 5}$ & 3.26332 & 3.59116 & $\mathbf{1 0 0 0}$ & 0.05638 & 0.05660 \\
$1 \mathrm{e}-8$ & $\mathbf{0 . 5 5}$ & 0.01876 & 0.01924 & $\mathbf{0 . 2 5}$ & 3.30041 & 3.65619 & $\mathbf{2 0 0}$ & 0.05619 & 0.05662 \\
\hline
\end{tabular}

Table 3: Minimum errors and corresponding stabilization parameter $\rho$ for $\left(P_{2}, P_{1}\right)$ element

\section{Acknowledgments}

The first author would like to express his gratitude to the Department of Science and Technology (DST), Government of India, for the financial support (DST/INSPIRE Fellowship/IF170401).

\section{Declarations}

\section{Data Availability Statement}

The datasets generated during and analysed during the current study are available from the corresponding author on reasonable request.

\section{Conflict of Interest}

The authors declare that they have no conflict of interest.

\section{References}

[1] Abbaszadeh, M. and Dehghan, M. Investigation of the Oldroyd model as a generalized incompressible Navier-Stokes equation via the interpolating stabilized element free Galerkin technique, Appl. Numer. Math. 150, 274-294, (2020).

[2] Adams, R.A. Sobolev spaces. Academic Press, New York-London (1975).

[3] Ahmed, N. On the grad-div stabilization for the steady Oseen and Navier-Stokes equations, Calcolo 54(1), 471-501, (2017).

[4] Bir, B. and Goswami, D. On a three step two-grid finite element method for the Oldroyd model of order one, ZAMM-J. Appl. Math. Mech. 101(11), e202000373, (2021).

[5] Bir, B., Goswami, D., and Pani, A.K. Backward Euler method for the equations of motion arising in Oldroyd model of order one with nonsmooth initial data, IMA J. Numer. Anal. (2021). https://doi.org/10.1093/imanum/drab072. 
[6] Bir, B., Goswami, D., and Pani, A.K. Finite element penalty method for the Oldroyd model of order one with non-smooth initial data, Comput. Methods Appl. Math. (2022). (accepted).

[7] Braack, M. and Burman, E. Local projection stabilization for the Oseen problem and its interpretation as a variational multiscale method, SIAM J. Numer. Anal. 43, 2544-2566, (2006).

[8] Brenner, S.C. and Scott, L.R. The Mathematical Theory of Finite Element Methods (Vol. 3), Springer, New York (2008).

[9] Brezzi, F. and Russo, A. Choosing bubbles for advection-diffusion problems, Math. Models Methods Appl. Sci. 4, 571-587, (1994).

[10] Brooks, A. and Hughes, T.J.R. Streamline upwind/Petrov-Galerkin formulation for convection dominated flows with particular emphasis on the incompressible Navier-Stokes equations, Comput. Methods Appl. Mech. Engrg. 32, 199-259, (1982).

[11] Burman, E. and Hansbo, P. Edge stabilization for Galerkin approximations of convection-diffusionreaction problems, Comput. Methods Appl. Mech. Engrg. 193, 1437-1453, (2004).

[12] Ciarlet, P. G. The finite element method for elliptic problems. North-Holland Publishing Co., Amsterdam, 1978.

[13] Dallmann, H., Arndt, D., and Lube, G. Local projection stabilization for the Oseen problem, IMA J. Numer. Anal. 36, 796-823, (2016).

[14] de Frutos, J., Garcia-Archilla, B., John, V., and Novo, J. Grad-div stabilization for the evolutionary Oseen problem with inf-sup stable finite elements, J. Sci. Comput. 66(3), 991-1024, (2016).

[15] de Frutos, J., Garcia-Archilla, B., John, V., and Novo, J. Analysis of the grad-div stabilization for the time-dependent Navier-Stokes equations with inf-sup stable finite elements, Adv. Comput. Math. 44, 195-225, (2018).

[16] Franca, L.P. and Hughes, T.J.R. Two classes of mixed finite element methods, Comput. Methods Appl. Mech. Engrg. 69(1), 89-129, (1988).

[17] Galvin, K.J., Linke, A., Rebholz, L.G., and Wilson, N.E. Stabilizing poor mass conservation in incompressible flow problems with large irrotational forcing and application to thermal convection, Comput. Methods Appl. Mech. Eng. 237(240), 166-176, (2012).

[18] Ghia, U., Ghia, K.N., Shin, C.T. High-Re solutions for incompressible flow using the Navier-Stokes equations and a multigrid method. J. Comput. Phys. 48, 387-411, (1982).

[19] Goswami, D. and Pani, A. K. A priori error estimates for semidiscrete finite element approximations to the equations of motion arising in Oldroyd fluids of order one, Int. J. Numer. Anal. Model. 8(2), 324-352, (2011).

[20] He, Y., Lin, Y., Shen, S. S. P., Sun, W., and Tait, R. Finite element approximation for the viscoelastic fluid motion problem, J. Comput. Appl. Math. 155(2), 201-222, (2003).

[21] Hecht, F. New development in freefem ++, J. Numer. Math. 20, 251-265, (2012).

[22] Heywood, J. G. and Rannacher, R. Finite element approximation of the nonstationary Navier-Stokes problem I: Regularity of solutions and second-order error estimates for spatial discretization, SIAM J. Numer. Anal. 19(2), 275-311, (1982).

[23] Heywood, J. G. and Rannacher, R. Finite element approximation of the nonstationary Navier-Stokes problem part IV: Error analysis for second-order time discretization, SIAM J. Numer. Anal. 27(2), 353-384, (1990).

[24] Hill, A. T. and Süli, E. Approximation of the global attractor for the incompressible Navier-Stokes equations, IMA J. Numer. Anal. 20(4), 633-667, (2000).

[25] Jenkins, E.W., John, V., Linke, A., and Rebholz, L.G. On the parameter choice in grad-div stabilization for the Stokes equations, Adv. Comput. Math. 40, 491-516, (2014). 
[26] John, V. and Kindl, A. Numerical studies of finite element variational multiscale methods for turbulent flow simulations, Comput. Methods Appl. Mech Engrg. 199(1316), 841-852, (2010).

[27] Liu, C. and Si, Z. An incremental pressure correction finite element method for the time-dependent Oldroyd flows, Appl. Math. Comput. 351, 99-115, (2019).

[28] Matthies, G., Lube, G., and Röhe, L. Some remarks on residual-based stabilisation of inf-sup stable discretisations of the generalised Oseen problem, Comput. Methods Appl. Math. 9(4), 368-390, (2009).

[29] Mohan, M.T. Deterministic and stochastic equations of motion arising in Oldroyd fluids of order one: existence, uniqueness, exponential stability and invariant measures, Stoch. Anal. Appl. 38, 1-61, (2020).

[30] Oldroyd, J. G. Non-Newtonian flow of liquids and solids. Rheology: Theory and Applications, Vol. I (F. R. Eirich, Ed.), Academic Press, New York, 653-682, (1956).

[31] Olshanskii, M.A. A low order Galerkin finite element method for the Navier-Stokes equations of steady incompressible flow: a stabilization issue and iterative methods, Comput. Methods Appl. Mech. Engrg. 191, 5515-5536, (2002).

[32] Olshanskii, M.A. and Reusken, A. Grad-div stabilization for Stokes equations, Math Comp. 73, 1699$1718(2004)$

[33] Olshanskii, M.A., Lube, G., Heister, T., and Lowe, J. Graddiv stabilization and subgrid pressure models for the incompressible NavierStokes equations, Comput. Methods Appl. Mech. Engrg. 198, 3975-3988, (2009).

[34] Pani, A.K. and Yuan, J.Y. Semidiscrete finite element Galerkin approximations to the equations of motion arising in the Oldroyd model, IMA J. Numer. Anal. 25(4), 750-782, (2005).

[35] Pani, A.K. , Yuan, J.Y., and Damazio, P. On a linearized backward Euler method for the equations of motion arising in the Oldroyd fluids of order one, SIAM J. Numer. Anal. 44(2), 804-825, (2006).

[36] Rohe, L., and Lube, G. Analysis of a variational multiscale method for large-eddy simulation and its application to homogeneous isotropic turbulence, Comput. Methods Appl. Mech. Engrg. 199(37-40), 2331$2342,(2010)$.

[37] Wang, K., He, Y., and Feng, X. On error estimates of the penalty method for the viscoelastic flow problem I: Time discretization, Appl. Math. Model. 34, 4089-4105, (2010).

[38] Wang, K., He, Y., and Shang, Y. Fully discrete finite element method for the viscoelastic fluid motion equations, Disc. Cont. Dyn. Sys. Ser. B 13(3), 665-684, (2010).

[39] Wang, K., He, Y., and Feng, X. On error estimates of the fully discrete penalty method for the viscoelastic flow problem, Int. J. Comput. Math. 88, 2199-2220, (2011).

[40] Yang, Y. Lei, Y., and Si, Z. Unconditional stability and error estimates of the modified characteristics FEM for the time-dependent viscoelastic Oldroyd flows, Adv. Appl. Math. Mech. 13(2), 311-332, (2020).

[41] Zhang, T. and Qian, Y. Stability analysis of several first order schemes for the Oldroyd model with smooth and nonsmooth initial data, Numer. Meth. Partial Diff. Eqn. 34(4), 2180-2216, (2018).

[42] Zhang, T. ,Qian, Y., Jiang, T., and Yuan, J. Stability and convergence of the higher projection method for the time-dependent viscoelastic flow problem, J. Comput. Appl. Math. 318(4), 1-21, (2018). 